\title{
A Stable High-Order Perturbation of Surfaces Method for Numerical Simulation of Diffraction Problems in Triply Layered Media
}

\author{
Youngjoon Hong and David P. Nicholls \\ Department of Mathematics, Statistics, and Computer Science, University of Illinois at \\ Chicago, Chicago, IL, 60607 U.S.A.
}

\begin{abstract}
The accurate numerical simulation of linear waves interacting with periodic layered media is a crucial capability in engineering applications. In this contribution we study the stable and high-order accurate numerical simulation of the interaction of linear, time-harmonic waves with a periodic, triply layered medium with irregular interfaces. In contrast with volumetric approaches, High-Order Perturbation of Surfaces (HOPS) algorithms are inexpensive interfacial methods which rapidly and recursively estimate scattering returns by perturbation of the interface shape. In comparison with Boundary Integral/Element Methods, the stable HOPS algorithm we describe here does not require specialized quadrature rules, periodization strategies, or the solution of dense non-symmetric positive definite linear systems. In addition, the algorithm is provably stable as opposed to other classical HOPS approaches. With numerical experiments we show the remarkable efficiency, fidelity, and accuracy one can achieve with an implementation of this algorithm.
\end{abstract}

Keywords: High-Order Spectral Methods, Time-Harmonic Linear Wave Scattering, Periodic Layered Media, High-Order Perturbation of Surfaces Methods 2000 MSC: 78A45, 65N35, 35J05, 41A58

Email address: hongy@uic.edu; davidn@uic.edu (Youngjoon Hong and David P. Nicholls)

Preprint submitted to Journal of Computational Physics

October 24, 2016

(C) 2016. This manuscript version is made available under the Elsevier user license

http://www.elsevier.com/open-access/userlicense/1.0/ 


\section{Introduction}

The accurate numerical simulation of linear waves interacting with periodic layered media is a crucial capability in many areas of scientific and industrial interest. Examples exist in areas such as geophysics [1,2], materials science [3], imaging [4], oceanography [5], and nanoplasmonics [6, 7, 8]. The latter includes topics as diverse as extraordinary optical transmission [9], surface enhanced spectroscopy [10], and surface plasmon resonance biosensing $[11,12,13,14,15,16]$. For each application, it is necessary to approximate the scattering returns of such models in a fast, highly accurate, and reliable fashion.

While all of the classical numerical algorithms (e.g., Finite Differences and Finite/Spectral Element methods) have been brought to bear upon this problem (see, particularly, the work of Dobson [17, 18] and Bao [19, 20, 21] on Finite Element solution of the doubly layered problem), we have recently argued $[22,23,24,25]$ that such volumetric approaches are greatly disadvantaged with an unnecessarily large number of unknowns for the layered media problems we consider here. Interfacial methods based upon Integral Equations (IEs) [26], for instance [27, 28, 29, 30, 31, 32], are a natural candidate but, as we have pointed out [22, 23, 24, 25], these also face difficulties. Most of these have been addressed in recent years through (i.) the use of sophisticated quadrature rules to deliver High-Order Spectral accuracy, (ii.) the design of preconditioned iterative solvers with suitable acceleration [33], and (iii.) new strategies to avoid periodizing the Green function [27, 28, 29, 30, 31, 32]. Consequently, they are a compelling alternative (see, e.g., the survey article of [34] for more details), however, two properties render them non-competitive for the parameterized problems we consider as compared with the methods we advocate here. First, for configurations characterized by the real value $\varepsilon$ (for us the heights/slopes of the irregular interfaces), an IE solver will return the scattering returns only for a particular value of $\varepsilon$. If this value is changed then the solver must be run again. Second, the dense, non-symmetric positive definite systems of linear equations generated by IEs which must be inverted with each simulation. We note that the "Rigorous Coupled Wave Analysis" (RCWA) [35, 36] is very popular amongst practitioners for the problem we consider here. While it is very convenient to code and can be very fast if implemented with care, non-trivial interface shapes are modeled as thin layers using a staircase approximation which is, necessarily, a low-order approximation. 
A "High Order Perturbation of Surfaces" (HOPS) approach can effectively address these concerns. More specifically, in [23, 24, 25] we put forth the method of Field Expansions (FE) which traces its roots to the low-order calculations of Rayleigh [37] and Rice [38]. This was extended to a high-order algorithm by Bruno \& Reitich [39, 40, 41] and later enhanced and stabilized by Nicholls and Reitich [42, 43, 44], and Nicholls and Malcolm [45, 23, 25]. These formulations are particularly compelling as they maintain the advantageous properties of classical IE formulations (e.g., surface formulation and exact enforcement of far-field and quasiperiodicity conditions) while avoiding the shortcomings listed above. First, since the methods are built upon expansions in the boundary parameter, $\varepsilon$, once the Taylor coefficients are known for the scattering quantities, it is simply a matter of summing these (rather than beginning a new simulation) for any given choice of $\varepsilon$ to recover the returns. Second, due to the nature of the scheme, at every perturbation order one need only invert a single, sparse operator corresponding to the flat-interface, order-zero approximation of the problem.

However, Nicholls and Reitich [46] showed that, like other classical HOPS schemes, the FE method depends upon strong cancellations for its convergence which can result in quite ill-conditioned simulations. We refer the interested reader to $[46,47,48,42,43]$ for the initial description of this phenomena, and the additional exhaustive and illuminating simulations of Wilkening and Vasan [49].

In response to these observations, Nicholls and Reitich described an alternative HOPS scheme, the method of Transformed Field Expansions (TFE), which does not possess strong cancellations [46, 47, 48, 42, 43]. In fact, the resulting recursions can be used in a rigorous proof of the strong convergence of the perturbation expansions in a Sobolev space $[46,48]$, which was later extended to Lipschitz profiles in [50]. In addition, the TFE recursions were implemented to reveal a stable and highly accurate numerical scheme for the simulation of scattering returns by singly layered periodic gratings [47, 43].

This work was generalized by Nicholls and Shen to the case of irregular bounded obstacles in two [51] and three dimensions [52], who later delivered a rigorous numerical analysis of the method [53]. Subsequently, He, Nicholls, and Shen [54] devised a highly non-trivial extension to the case of periodic gratings separating two materials of different dielectric constants. Here, of course, one must be concerned not only with a reflected field and its far-field boundary condition (upward propagating) at positive infinity, but also with a transmitted field which satisfies a different condition (downward propagating) 
at negative infinity. In this contribution we make the further extension to the case of three layers which introduces the added complication of waves propagating both "up" and "down" in a vertically bounded layer in between. This difficulty manifests itself in the governing equations (and our numerical algorithm) through the complication of a coupled system of three boundary value problems (at each wavenumber in the spatial variable).

More specifically, to begin we introduce artificial boundaries above and below the interfaces of the structure, which truncate the unbounded domain. We use Dirichlet-Neumann operators at each of these artificial boundaries to enforce the outgoing wave conditions transparently and without reflection $[55,56,57,58,59,60,61,62,63,64]$. Our spectrally accurate method involves the novelty of a modified Legendre-Galerkin approach where the standard basis is supplemented with additional connecting basis functions across the layer boundaries in the spirit of the work by He, Nicholls, and Shen [54] for two layers. However, our contribution is more subtle than what appears in the aforementioned publication; for more details we refer the reader to Section 5 .

The organization of the paper is as follows: In Section 2 we recall the governing equations of an electromagnetic field incident upon a periodic, twodimensional, triply layered irregular grating structure. In Section 3 we derive the TFE recursions in this triply layered context, and discuss a LegendreGalerkin method to solve the resulting coupled boundary value problems in Sections 4 and 5. Extensive numerical results are provided in Section 6, followed by concluding remarks in Section 7 .

\section{Governing Equations}

The geometry we consider is displayed in Figure 1: A $z$-invariant, triply layered structure. Dielectrics occupy all three domains, the upper (with refractive index $n^{u}$ ) fills the region

$$
S_{g}^{u}:=\{y>\bar{g}+g(x)\},
$$

the middle (with refractive index $n^{v}$ ) occupies

$$
S_{g, h}^{v}:=\{\bar{h}+h(x)<y<\bar{g}+g(x)\},
$$

while the lower (with index of refraction $n^{w}$ ) fills

$$
S_{h}^{w}:=\{z<\bar{h}+h(x)\} .
$$




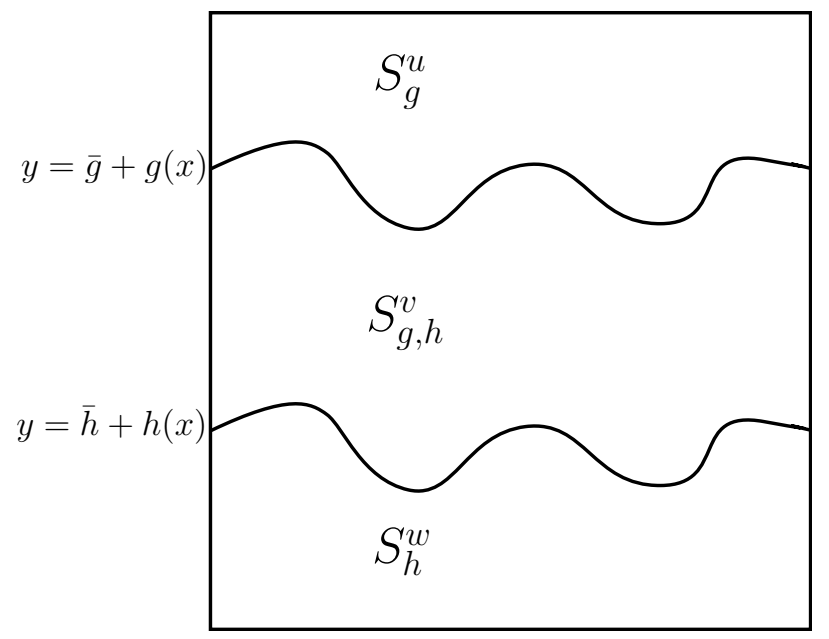

Figure 1: A depiction of the triply layered grating structure with layer interfaces at $y=$ $\bar{g}+g(x)$ and $y=\bar{h}+h(x)$.

The gratings are $d$-periodic so that $g(x+d)=g(x)$ and $h(x+d)=h(x)$. The structure is illuminated from above by monochromatic plane-wave incident radiation of frequency $\omega$, aligned with the grooves

$$
\underline{\mathbf{E}}^{i n c}(x, y, t)=\mathbf{A} e^{\mathrm{i} \alpha x-\mathrm{i} \beta^{u} y-\mathrm{i} \omega t}, \quad \underline{\mathbf{H}}^{i n c}(x, y, t)=\mathbf{B} e^{\mathrm{i} \alpha x-\mathrm{i} \beta^{u} y-\mathrm{i} \omega t} .
$$

We consider the reduced electric and magnetic fields

$$
\mathbf{E}(x, y)=e^{\mathrm{i} \omega t} \underline{\mathbf{E}}(x, y, t), \quad \mathbf{H}(x, y)=e^{\mathrm{i} \omega t} \underline{\mathbf{H}}(x, y, t),
$$

which, like the reduced scattered fields, are $\alpha$-quasiperiodic due to the incident radiation. To close the problem, we specify that the scattered radiation is "outgoing" (upward propagating in $S_{g}^{u}$ and downward propagating in $S_{h}^{w}$ ).

It is well known (see, e.g., Petit [65]) that in this two-dimensional setting, the time-harmonic Maxwell equations decouple into two scalar Helmholtz problems which govern the transverse electric (TE) and transverse magnetic (TM) polarizations. We define the invariant $(z)$ direction of the scattered (electric or magnetic) fields by

$$
u=u(x, y), \quad v=v(x, y), \quad w=w(x, y),
$$

in $S_{g}^{u}, S_{g, h}^{v}$, and $S_{h}^{w}$, respectively. The incident radiation in the upper layer is specified by $u^{\text {inc }}$. 
We find that we must seek outgoing, $\alpha$-quasiperiodic solutions of

$$
\begin{array}{ll}
\Delta u+k_{u}^{2} u=0, & \text { in } S_{g}^{u}, \\
\Delta v+k_{v}^{2} v=0, & \text { in } S_{g, h}^{v}, \\
\Delta w+k_{w}^{2} w=0, & \text { in } S_{h}^{w}, \\
u-v=-u^{i n c}, & y=\bar{g}+g(x), \\
v-w=0, & y=\bar{h}+h(x), \\
\partial_{N_{g}} u-\tau^{2} \partial_{N_{g}} v=-\partial_{N_{g}} u^{i n c}, & y=\bar{g}+g(x), \\
\partial_{N_{h}} v-\sigma^{2} \partial_{N_{h}} w=0, & y=\bar{h}+h(x),
\end{array}
$$

where $k_{m}=n^{m} \omega / c_{0}=\omega / c^{m}, m \in\{u, v, w\}, c_{0}$ is the speed of light, $N_{g}=$ $\left(-\partial_{x} g, 1\right)^{T}, N_{h}=\left(-\partial_{x} h, 1\right)^{T}$, and

$$
\tau^{2}= \begin{cases}1 & \mathrm{TE} \\ \left(k_{u} / k_{v}\right)^{2}=\left(n^{u} / n^{v}\right)^{2} & \mathrm{TM}\end{cases}
$$

and

$$
\sigma^{2}= \begin{cases}1 & \mathrm{TE} \\ \left(k_{v} / k_{w}\right)^{2}=\left(n^{v} / n^{w}\right)^{2} & \mathrm{TM} .\end{cases}
$$

106

\subsection{Transparent Boundary Conditions}

Following the standard TFE methodology $[47,43,54]$ we introduce artificial boundaries and transparent boundary conditions to solve (1) (see also the work of Dobson [17, 18], Bao [19, 20, 21], and He, Min, and Nicholls [66] for such developments in the setting of Finite/Spectral Element solution of layered medium problems). More precisely, we introduce the planes $y=a>\bar{g}+|g|_{L^{\infty}}$ and $y=b<\bar{h}-|h|_{L^{\infty}}$, and define the domains

$$
\begin{aligned}
S^{a}:=\{y>a\}, & S^{b}:=\{y<b\}, \\
S_{g}^{a, u}:=\{\bar{g}+g(x)<y<a\}, & S_{h}^{w, b}:=\{b<y<\bar{h}+h(x)\} ;
\end{aligned}
$$

107

see Figure 2.

The transparent boundary conditions are based upon the Rayleigh expansions [37] which can be used to solve the problems on $S^{a}$ and $S^{b}$ explicitly with generic Dirichlet data, say $\zeta(x)$ and $\psi(x)$, at the artificial boundaries $\{y=a\}$ and $\{y=b\}$, respectively. More precisely we have

$$
u=u(x, y)=\sum_{p=-\infty}^{\infty} \hat{\zeta}_{p} e^{\mathrm{i} \alpha_{p} x+\mathrm{i} \beta_{p}^{u}(y-a)}, \quad y>a,
$$




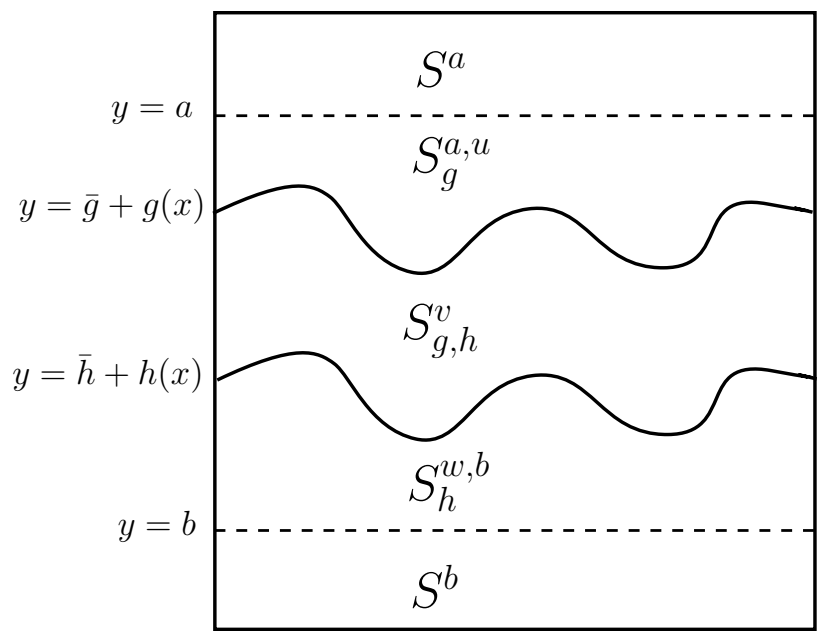

Figure 2: A depiction of the truncated triply layered grating structure with layer interfaces at $y=\bar{g}+g(x)$ and $y=\bar{h}+h(x)$, and artificial boundaries at $y=a$ and $y=b$.

and

$$
w=w(x, y)=\sum_{p=-\infty}^{\infty} \hat{\psi}_{p} e^{\mathrm{i} \alpha_{p} x-\mathrm{i} \beta_{p}^{w}(y-b)}, \quad y<b,
$$

where, for $p \in \mathbf{Z}$ and $m \in\{u, w\}$,

$$
\alpha_{p}:=\alpha+(2 \pi / d) p, \quad \beta_{p}^{m}:=\left\{\begin{array}{ll}
\sqrt{\left(k_{m}\right)^{2}-\alpha_{p}^{2}} & p \in \mathcal{U}^{m} \\
\mathrm{i} \sqrt{\alpha_{p}^{2}-\left(k_{m}\right)^{2}} & p \notin \mathcal{U}^{m}
\end{array},\right.
$$

and the set of propagating modes is given by

$$
\mathcal{U}^{m}:=\left\{p \in \mathbf{Z} \mid \alpha_{p}^{2}<\left(k_{m}\right)^{2}\right\} .
$$

Notice that

$$
u(x, a)=\sum_{p=-\infty}^{\infty} \hat{\zeta}_{p} e^{\mathrm{i} \alpha_{p} x}=\zeta(x), \quad w(x, b)=\sum_{p=-\infty}^{\infty} \hat{\psi}_{p} e^{\mathrm{i} \alpha_{p} x}=\psi(x) .
$$

With these solution formulas we can compute the Neumann data at the artificial boundaries

$$
\partial_{y} u(x, a)=\sum_{p=-\infty}^{\infty}\left(\mathrm{i} \beta_{p}^{u}\right) \hat{\zeta}_{p} e^{\mathrm{i} \alpha_{p} x}, \quad \partial_{y} w(x, b)=\sum_{p=-\infty}^{\infty}\left(-\mathrm{i} \beta_{p}^{w}\right) \hat{\psi}_{p} e^{\mathrm{i} \alpha_{p} x}
$$


which permit us to define the Dirichlet-Neumman Operators (DNOs)

$$
T_{1}[\zeta]:=\sum_{p=-\infty}^{\infty}\left(\mathrm{i} \beta_{p}^{u}\right) \hat{\zeta}_{p} e^{\mathrm{i} \alpha_{p} x}, \quad T_{3}[\psi]:=\sum_{p=-\infty}^{\infty}\left(-\mathrm{i} \beta_{p}^{w}\right) \hat{\psi}_{p} e^{\mathrm{i} \alpha_{p} x}
$$

108

which are order-one Fourier multipliers.

Using these we can state (1) equivalently on the bounded domain $\{b<$ $y<a\}$ as

$$
\begin{array}{ll}
\Delta u+k_{u}^{2} u=0, & \text { in } S_{g}^{a, u}, \\
\Delta v+k_{v}^{2} v=0, & \text { in } S_{g, h}^{v}, \\
\Delta w+k_{w}^{2} w=0, & \text { in } S_{h}^{w, b}, \\
u-v=-u^{i n c}, & y=\bar{g}+g(x), \\
v-w=0, & y=\bar{h}+h(x), \\
\partial_{N_{g}} u-\tau^{2} \partial_{N_{g}} v=-\partial_{N_{g}} u^{i n c}, & y=\bar{g}+g(x), \\
\partial_{N_{h}} v-\sigma^{2} \partial_{N_{h}} w=0, & y=\bar{h}+h(x), \\
\partial_{y} u-T_{1}[u]=0, & y=a, \\
\partial_{y} w-T_{3}[w]=0, & y=b,
\end{array}
$$

which we use as the starting point for our numerical method.

\section{Transformed Field Expansions}

In this section, we present the Transformed Field Expansion (TFE) algorithm. First, we introduce a domain flattening change of variables known as $\sigma$-coordinates [67] in the oceanographic literature, and the C-method [68] in the electromagnetics community. Then, we consider a boundary perturbation algorithm to solve the resulting equations which leads to the provably convergent TFE recursions. 
117

\subsection{The Change of variables}

We define the change of variables:

$$
\begin{array}{ll}
x^{\prime}=x, & \\
y_{1}=a\left(\frac{y-(\bar{g}+g)}{a-(\bar{g}+g)}\right)+\bar{g}\left(\frac{a-y}{a-(\bar{g}+g)}\right), & \bar{g}+g<y<a, \\
y_{2}=\bar{h}\left(\frac{y-(\bar{g}+g)}{(\bar{h}+h)-(\bar{g}+g)}\right)+\bar{g}\left(\frac{(\bar{h}+h)-y}{(\bar{h}+h)-(\bar{g}+g)}\right), & \bar{h}+h<y<\bar{g}+g, \\
y_{3}=b\left(\frac{y-(\bar{h}+h)}{b-(\bar{h}+h)}\right)+\bar{h}\left(\frac{b-y}{b-(\bar{h}+h)}\right) & b<y<\bar{h}+h,
\end{array}
$$

which maps

$$
S_{g}^{a, u} \rightarrow S_{0}^{a, u}, \quad S_{g, h}^{v} \rightarrow S_{0,0}^{v}, \quad S_{h}^{w, b} \rightarrow S_{0}^{w, b}
$$

We note that

$$
b \leq y_{3} \leq \bar{h} \leq y_{2} \leq \bar{g} \leq y_{1} \leq a .
$$

A long calculation, presented in Appendix A, shows that (2) transforms under this change of variables to the following system of equations to be solved.

$$
\begin{array}{ll}
\Delta_{x^{\prime}, y_{1}} u+k_{u}^{2} u=\mathcal{R}_{1}, & \text { in } S_{0}^{a, u}, \\
\Delta_{x^{\prime}, y_{2}} v+k_{v}^{2} v=\mathcal{R}_{2}, & \text { in } S_{0,0}^{v}, \\
\Delta_{x^{\prime}, y_{3}} w+k_{w}^{2} w=\mathcal{R}_{3}, & \text { in } S_{0}^{w, b}, \\
u-v=-e^{\mathrm{i} \alpha x^{\prime}} e^{-\mathrm{i} \beta^{u}(\bar{g}+g)}, & y_{1}=y_{2}=\bar{g}, \\
v-w=0, & y_{2}=y_{3}=\bar{h}, \\
\partial_{y_{1}} u-\tau^{2} \partial_{y_{2}} v=\mathcal{J}_{1}, & y_{1}=y_{2}=\bar{g}, \\
\partial_{y_{2}} v-\sigma^{2} \partial_{y_{3}} w=\mathcal{J}_{2}, & y_{2}=y_{3}=\bar{h}, \\
\partial_{y_{1}} u-T_{1}[u]=-\frac{g}{a-\bar{g}} T_{1}[u], & y_{1}=a, \\
\partial_{y_{3}} w-T_{3}[w]=-\frac{h}{b-\bar{h}} T_{3}[w], &
\end{array}
$$

where

$$
\mathcal{R}_{i}=\frac{1}{E_{i}^{2}}\left[\partial_{x^{\prime}} R_{i}^{x}+\partial_{y_{i}} R_{i}^{x}+R_{i}\right]
$$

118 We refer the reader to Appendix A for the particular forms for the right hand sides $\left\{\mathcal{R}_{1}, \mathcal{R}_{2}, \mathcal{R}_{3}, \mathcal{J}_{1}, \mathcal{J}_{2}\right\}$. 


\subsection{Boundary Perturbation}

We now consider a boundary perturbation method to solve the transformed governing equations, (3). To begin, we expand the fields in power series

$$
\{u, v, w\}=\sum_{n=0}^{\infty}\left\{u_{n}, v_{n}, w_{n}\right\}(x, y) \varepsilon^{n} .
$$

Inserting these expansions into (3) and equating at order $\mathcal{O}\left(\varepsilon^{n}\right)$ delivers

$$
\begin{array}{ll}
\Delta_{x^{\prime}, y_{1}} u_{n}+k_{u}^{2} u_{n}=\mathcal{R}_{1, n}, & \text { in } S_{0}^{a, u}, \\
\Delta_{x^{\prime}, y_{2}} v_{n}+k_{v}^{2} v_{n}=\mathcal{R}_{2, n}, & \text { in } S_{0,0}^{v}, \\
\Delta_{x^{\prime}, y_{3}} w_{n}+k_{w}^{2} w_{n}=\mathcal{R}_{3, n}, & \text { in } S_{0}^{w, b}, \\
u_{n}-v_{n}=-e^{\mathrm{i} \alpha x^{\prime}} e^{-\mathrm{i} \beta \bar{g}} \frac{\left(-\mathrm{i} \beta f_{g}\right)^{n}}{n !}, & y_{1}=y_{2}=\bar{g}, \\
v_{n}-w_{n}=0, & y_{2}=y_{3}=\bar{h}, \\
\partial_{y_{1}} u_{n}-\tau^{2} \partial_{y_{2}} v_{n}=\mathcal{J}_{1, n}, & y_{1}=y_{2}=\bar{g}, \\
\partial_{y_{2}} v_{n}-\sigma^{2} \partial_{y_{3}} w_{n}=\mathcal{J}_{2, n}, & y_{2}=y_{3}=\bar{h}, \\
\partial_{y_{1}} u_{n}-T_{1}\left[u_{n}\right]=-\frac{f_{g}}{a-\bar{g}} T_{1}\left[u_{n-1}\right], & y_{1}=a, \\
\partial_{y_{3}} w_{n}-T_{3}\left[w_{n}\right]=-\frac{f_{h}}{b-\bar{h}} T_{3}\left[w_{n-1}\right], & y_{3}=b .
\end{array}
$$

In these we have defined

$$
\mathcal{R}_{i, n}=\frac{1}{E_{i}^{2}}\left[\partial_{x^{\prime}} R_{i, n}^{x}+\partial_{y_{i}} R_{i, n}^{x}+R_{i, n}\right]
$$

121 and the remaining terms are specified in Appendix A.

Using the quasiperiodicity of the solutions, we make the following (generalized) Fourier series expansions,

$$
\begin{gathered}
\left\{u_{n}, v_{n}, w_{n}\right\}=\sum_{p=-\infty}^{\infty}\left\{u_{n}^{p}(y), v_{n}^{p}(y), w_{n}^{p}(y)\right\} e^{\mathrm{i} \alpha_{p} x} \\
\mathcal{R}_{i, n}(x, y)=\sum_{p=-\infty}^{\infty} \mathcal{R}_{i, n}^{p}(y) e^{\mathrm{i} \alpha_{p} x}, \quad \mathcal{J}_{i, n}(x)=\sum_{p=-\infty}^{\infty} \mathcal{J}_{i, n}^{p} e^{\mathrm{i} \alpha_{p} x},
\end{gathered}
$$


for $i=1,2,3$. Insertion of these into (4) delivers

$$
\begin{array}{ll}
\partial_{y}^{2} u_{n}^{p}(y)+\left(k_{u}^{2}-\alpha_{p}^{2}\right) u_{n}^{p}(y)=\mathcal{R}_{1, n}^{p}(y), & \bar{g}<y<a, \\
\partial_{y}^{2} v_{n}^{p}(y)+\left(k_{v}^{2}-\alpha_{p}^{2}\right) v_{n}^{p}(y)=\mathcal{R}_{2, n}^{p}(y), & \bar{h}<y<\bar{g}, \\
\partial_{y}^{2} w_{n}^{p}(y)+\left(k_{w}^{2}-\alpha_{p}^{2}\right) w_{n}^{p}(y)=\mathcal{R}_{3, n}^{p}(y), & b<y<\bar{h}, \\
u_{n}^{p}(\bar{g})-v_{n}^{p}(\bar{g})=-e^{-\mathrm{i} \beta \bar{g}} \frac{\left(-\mathrm{i} \beta f_{g}\right)^{n}}{n !}=: \zeta_{n}^{p}, & \\
v_{n}^{p}(\bar{h})-w_{n}^{p}(\bar{h})=0, & \\
\partial_{y} u_{n}^{p}(\bar{g})-\tau^{2} \partial_{y} v_{n}^{p}(\bar{g})=\mathcal{J}_{1, n}^{p}, & \\
\partial_{y} v_{n}^{p}(\bar{h})-\sigma^{2} \partial_{y} w_{n}^{p}(\bar{h})=\mathcal{J}_{2, n}^{p}, & y=a, \\
\partial_{y} u_{n}^{p}-\mathrm{i} \beta_{p}^{u} u_{n}^{p}=-\frac{f_{g}}{a-\bar{g}} T_{1}^{p}\left[u_{n-1}^{p}\right]=: \mathcal{B}_{1, n}^{p}, & \\
\partial_{y} w_{n}^{p}+\mathrm{i} \beta_{p}^{w} w_{n}^{p}=-\frac{f_{h}}{b-\bar{h}} T_{2}^{p}\left[w_{n-1}^{p}\right]=: \mathcal{B}_{2, n}^{p}, & y=b .
\end{array}
$$

\section{A Weak formulation of the Boundary Value Problem}

In order to homogenize (5) for our weak formulation we decompose the solutions $\left\{u_{n}^{p}, v_{n}^{p}, w_{n}^{p}\right\}$ as

$$
\left\{u_{n}^{p}, v_{n}^{p}, w_{n}^{p}\right\}=\left\{\tilde{u}_{n}^{p}, \tilde{v}_{n}^{p}, \tilde{w}_{n}^{p}\right\}+\left\{\check{u}_{n}^{p}, \check{v}_{n}^{p}, \check{w}_{n}^{p}\right\},
$$

where $\left\{\tilde{u}_{n}^{p}, \tilde{v}_{n}^{p}, \tilde{w}_{n}^{p}\right\}$ solve (5) with $\mathcal{R}_{i, n}^{p} \equiv 0$, while $\left\{\check{u}_{n}^{p}, \check{v}_{n}^{p}, \check{w}_{n}^{p}\right\}$ solve (5) with $\mathcal{B}_{i, n}^{p} \equiv \zeta_{n}^{p} \equiv \mathcal{J}_{i, n}^{p} \equiv 0$. More specifically, dropping the $\{n, p\}$ indices, we write

$$
\begin{array}{ll}
\partial_{y}^{2} \tilde{u}(y)+\left(k_{u}^{2}-\alpha^{2}\right) \tilde{u}(y)=0, & \bar{g}<y<a \\
\partial_{y}^{2} \tilde{v}(y)+\left(k_{v}^{2}-\alpha^{2}\right) \tilde{v}(y)=0, & \bar{h}<y<\bar{g} \\
\partial_{y}^{2} \tilde{w}(y)+\left(k_{w}^{2}-\alpha^{2}\right) \tilde{w}(y)=0, & b<y<\bar{h} \\
\tilde{u}(\bar{g})-\tilde{v}(\bar{g})=\zeta, & \\
\tilde{v}(\bar{h})-\tilde{w}(\bar{h})=0, & \\
\partial_{y} \tilde{u}(\bar{g})-\tau^{2} \partial_{y} \tilde{v}(\bar{g})=\mathcal{J}_{1}, & \\
\partial_{y} \tilde{v}(\bar{h})-\sigma^{2} \partial_{y} \tilde{w}(\bar{h})=\mathcal{J}_{2}, & y=a, \\
\partial_{y} \tilde{u}-\mathrm{i} \beta^{u} \tilde{u}=\mathcal{B}_{1}, & y=b . \\
\partial_{y} \tilde{w}+\mathrm{i} \beta^{w} \tilde{w}=\mathcal{B}_{2}, & y
\end{array}
$$


Due to their simplicity, (6) can be solved explicitly so it remains to solve for $\{\check{u}, \check{v}, \check{w}\}$ which we accomplish by a High-Order Spectral method. For the latter we set

$$
\begin{gathered}
\tilde{u}=M_{1} e^{\mathrm{i} \beta^{u} y}+N_{1} e^{-\mathrm{i} \beta^{u} y}, \quad \tilde{v}=M_{2} e^{\mathrm{i} \beta^{v} y}+N_{2} e^{-\mathrm{i} \beta^{v} y}, \\
\tilde{w}=M_{3} e^{\mathrm{i} \beta^{w} y}+N_{3} e^{-\mathrm{i} \beta^{w} y}
\end{gathered}
$$

which we substitute into (6), and then find explicit forms for $M_{i}$ and $N_{i}$.

For $\left\{\check{u}_{n}^{p}, \check{v}_{n}^{p}, \check{w}_{n}^{p}\right\}$ we consider the following equations

$$
\begin{array}{ll}
\partial_{y}^{2} \check{u}(y)+\left(k_{u}^{2}-\alpha^{2}\right) \check{u}(y)=\mathcal{R}_{1}(y), & \bar{g}<y<a \\
\partial_{y}^{2} \check{v}(y)+\left(k_{v}^{2}-\alpha^{2}\right) \check{v}(y)=\mathcal{R}_{2}(y), & \bar{h}<y<\bar{g} \\
\partial_{y}^{2} \check{w}(y)+\left(k_{w}^{2}-\alpha^{2}\right) \check{w}(y)=\mathcal{R}_{3}(y), & b<y<\bar{h} \\
\check{u}(\bar{g})-\check{v}(\bar{g})=0, & \\
\check{v}(\bar{h})-\check{w}(\bar{h})=0, & \\
\partial_{y} \check{u}(\bar{g})-\tau^{2} \partial_{y} \check{v}(\bar{g})=0, & \\
\partial_{y} \check{v}(\bar{h})-\sigma^{2} \partial_{y} \check{w}(\bar{h})=0, & y=a, \\
\partial_{y} \check{u}-\mathrm{i} \beta^{u} \check{u}=0, & y=b . \\
\partial_{y} \check{w}+\mathrm{i} \beta^{w} \check{w}=0, &
\end{array}
$$

Our weak formulation of $(7)$ is: Find $U \in H^{1}([b, a])$ such that

$$
\begin{aligned}
\left(\tilde{k}^{2} U, \varphi\right)-\left(\partial_{y} U, \partial_{y} \varphi\right)+\left(1-\tau^{2}\right) \partial_{y} v(\bar{g}) \bar{\varphi}(\bar{g})+\left(\sigma^{-2}-1\right) \partial_{y} v(\bar{h}) \bar{\varphi}(\bar{h}) \\
=(\mathcal{R}, \varphi)-\mathrm{i} \beta_{u} u(a) \bar{\varphi}(a)-\mathrm{i} \beta_{w} w(b) \bar{\varphi}(b), \quad \forall \varphi \in H^{1}([b, a])
\end{aligned}
$$

where

$$
\begin{gathered}
U=\left\{\begin{array}{ll}
u_{N}, & y \in I_{1}, \\
v_{N}, & y \in I_{2}, \\
w_{N}, & y \in I_{3},
\end{array} \quad \varphi= \begin{cases}\varphi_{1}, & y \in I_{1}, \\
\varphi_{2}, & y \in I_{2}, \\
\varphi_{3}, & y \in I_{3},\end{cases} \right. \\
\mathcal{R}=\left\{\begin{array}{ll}
\mathcal{R}_{1}, & y \in I_{1}, \\
\mathcal{R}_{2}, & y \in I_{2}, \\
\mathcal{R}_{3}, & y \in I_{3},
\end{array} \quad \tilde{k}= \begin{cases}k_{u}, & y \in I_{1}, \\
k_{v}, & y \in I_{2}, \\
k_{w}, & y \in I_{3},\end{cases} \right.
\end{gathered}
$$

and

$$
I_{1}:=\{\bar{g}<y<a\}, \quad I_{2}:=\{\bar{h}<y<\bar{g}\}, \quad I_{3}:=\{b<y<\bar{h}\} .
$$


Following He, Nicholls, and Shen [54], we define the following finite dimensional function space $X_{N_{y}} \subset H^{1}([b, a])$ to specify a Legendre-Galerkin method,

$$
X_{N_{y}}:=\left\{\varphi_{i} \in P_{N_{y}}\left(I_{i}\right) \mid \partial_{y} \varphi_{1}(a)-\mathrm{i} \beta^{u} \varphi_{1}(a)=0, \partial_{y} \varphi_{3}(b)+\mathrm{i} \beta^{w} \varphi_{3}(b)=0\right\},
$$

where $P_{N_{y}}$ is the space of polynomials of degree less than $N_{y}$. The LegendreGalerkin formulation becomes: Find $U_{N_{y}} \in X_{N_{y}}$ such that

$$
\begin{aligned}
\left(\tilde{k}^{2} U_{N_{y}}, \varphi\right)- & \left(\partial_{y} U_{N_{y}}, \partial_{y} \varphi\right)+\left(1-\tau^{2}\right) \partial_{y} v_{N_{y}}(\bar{g}) \bar{\varphi}(\bar{g})+\left(\sigma^{-2}-1\right) \partial_{y} v_{N_{y}}(\bar{h}) \bar{\varphi}(\bar{h}) \\
& =\left(I_{N_{y}} f, \varphi\right)-\mathrm{i} \beta^{u} u_{N_{y}}(a) \bar{\varphi}(a)-\mathrm{i} \beta^{w} u_{N_{y}}(b) \bar{\varphi}(b), \quad \forall \varphi \in X_{N_{y}} .
\end{aligned}
$$

where $I_{N_{y}}$ is the projection operator onto $P_{N_{y}}$. Using integration-by-parts on each subdomain, we can obtain the alternate variational formulation: Find $U_{N_{y}} \in X_{N_{y}}$ such that

$$
\begin{aligned}
\left(\partial_{y}^{2} U_{N_{y}}, \varphi\right) & +\tilde{k}^{2}\left(U_{N_{y}}, \varphi\right)+\partial_{y} u_{N_{y}}(\bar{g}) \bar{\varphi}(\bar{g})-\tau^{2} \partial_{y} v_{N_{y}}(\bar{g}) \bar{\varphi}(\bar{g}) \\
& +\sigma^{-2} \partial_{y} v_{N_{y}}(\bar{h}) \bar{\varphi}(\bar{h})-\partial_{y} w_{N_{y}}(\bar{h}) \bar{\varphi}(\bar{h})=\left(I_{N_{y}} \mathcal{R}, \varphi\right), \quad \forall \varphi \in X_{N_{y}} .
\end{aligned}
$$

\section{The Legendre-Galerkin Numerical Method}

At this point we follow the spectral Legendre-Galerkin approach of Shen $[69,70,54]$ and consider as basis functions combinations of Legendre polynomials, $L_{j}(s)$. For $y \in I_{1}$ we define

$$
\begin{aligned}
\phi_{1, j}:=(1+\mathrm{i}) L_{j}\left(\frac{2(y-a)}{a-\bar{g}}+1\right)+a_{1, j} L_{j+1} & \left(\frac{2(y-a)}{a-\bar{g}}+1\right) \\
& +b_{1, j} L_{j+2}\left(\frac{2(y-a)}{a-\bar{g}}+1\right),
\end{aligned}
$$

such that

$$
\left(\partial_{y} \phi_{1, j}-\mathrm{i} \beta^{u} \phi_{1, j}\right)(a)=0, \quad \phi_{1, j}(\bar{g})=0,
$$

for $j=1,2, \ldots, N_{y}-2$. Further, for $y \in I_{2}$, we denote

$$
\begin{aligned}
\phi_{2, j}:=(1+\mathrm{i}) L_{j}\left(\frac{2(y-\bar{g})}{\bar{g}-\bar{h}}+1\right)+a_{2, j} L_{j+1} & \left(\frac{2(y-\bar{g})}{\bar{g}-\bar{h}}+1\right) \\
& +b_{2, j} L_{j+2}\left(\frac{2(y-\bar{g})}{\bar{g}-\bar{h}}+1\right),
\end{aligned}
$$


where

$$
\phi_{2, j}(\bar{g})=0, \quad \phi_{2, j}(\bar{h})=0 .
$$

In a similar way we define, for $y \in I_{3}$,

$$
\begin{aligned}
\phi_{3, j}:=(1+\mathrm{i}) L_{j}\left(\frac{2(y-\bar{h})}{\bar{h}-b}+1\right)+a_{3, j} L_{j+1} & \left(\frac{2(y-\bar{h})}{\bar{h}-b}+1\right) \\
& +b_{3, j} L_{j+2}\left(\frac{2(y-\bar{h})}{\bar{h}-b}+1\right),
\end{aligned}
$$

where

$$
\partial_{y} \phi_{3, j}(b)+\mathrm{i} \beta^{w} \phi_{3, j}(b)=0, \quad \phi_{3, j}(\bar{h})=0 .
$$

Note that these basis functions vanish at the mean interface levels $y=\bar{g}$ and $y=\bar{h}$. For this reason (c.f., [54]), we introduce additional basis functions which are nonzero at $y=\bar{g}$ and $y=\bar{h}$ :

$$
\eta_{1}(y)= \begin{cases}c_{1}(y-\bar{g})+1, & \bar{g} \leq y \leq a \\ c_{2}(y-\bar{g})+1, & \bar{h} \leq y \leq \bar{g} \\ 0, & b \leq y \leq \bar{h}\end{cases}
$$

and

$$
\eta_{2}(y)= \begin{cases}0, & \bar{g} \leq y \leq a \\ c_{3}(y-\bar{h})+1, & \bar{h} \leq y \leq \bar{g} \\ c_{4}(y-\bar{h})+1, & b \leq y \leq \bar{h}\end{cases}
$$

chosen such that

$$
\begin{array}{ll}
\left(\partial_{y} \eta_{1}-\mathrm{i} \beta^{u} \eta_{1}\right)(a)=0, & \eta_{1}(\bar{h})=0 \\
\left(\partial_{y} \eta_{2}+\mathrm{i} \beta^{w} \eta_{2}\right)(b)=0, & \eta_{2}(\bar{g})=0 .
\end{array}
$$

It is easy to show that

$$
c_{1}=\frac{\mathrm{i} \beta^{u}}{1-\mathrm{i} \beta^{u}(a-\bar{g})}, \quad c_{2}=\frac{1}{\bar{g}-\bar{h}}, \quad c_{3}=\frac{1}{\bar{h}-\bar{g}}, \quad c_{4}=-\frac{\mathrm{i} \beta^{w}}{1+\mathrm{i} \beta^{w}(b-\bar{h})} .
$$

With these we define the basis functions defined on all $\{b<y<a\}$

$$
\tilde{\phi}_{j}(y)=\left\{\begin{array}{ll}
\phi_{1, j}(y), & \bar{g}<y<a, \\
0, & b<y<\bar{g},
\end{array} \quad j=0, \ldots, N_{y}-2\right.
$$


and

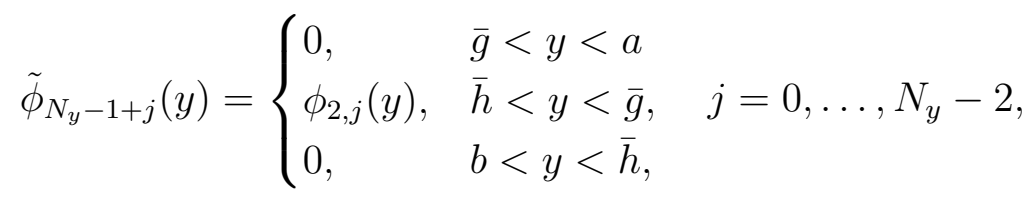

and

$$
\tilde{\phi}_{2 N_{y}-2+j}(y)=\left\{\begin{array}{ll}
0, & \bar{h}<y<a \\
\phi_{3, j}(y), & b<y<\bar{h},
\end{array} \quad j=0, \ldots, N_{y}-2,\right.
$$

and, finally,

$$
\tilde{\phi}_{3 N_{y}-3}(y)=\eta_{1}(y), \quad \tilde{\phi}_{3 N_{y}-2}(y)=\eta_{2}(y) .
$$

Our numerical approximation proceeds by seeking

$$
u^{N_{y}}(y):=\sum_{j=0}^{3 N_{y}-2} \hat{u}_{j} \tilde{\phi}_{j}(y)
$$

and setting

$$
\begin{aligned}
& \mathbf{u}=\left(\hat{u}_{0}, \ldots, \hat{u}_{N_{y}-2}\right)^{T}, \\
& \mathbf{v}=\left(\hat{u}_{N_{y}-1}, \ldots, \hat{u}_{2 N_{y}-3}\right)^{T}, \\
& \mathbf{w}=\left(\hat{u}_{2 N_{y}-2}, \ldots, \hat{u}_{3 N_{y}-4}\right)^{T}, \\
& \mathbf{f}=\left(\hat{f}_{0}, \ldots, \hat{f}_{3 N_{y}-4}\right)^{T},
\end{aligned}
$$

where

$$
\hat{f}_{j}:=\left(I_{N_{y}} f, \tilde{\phi}_{j}\right), \quad j=0, \ldots, 3 N_{y}-2 .
$$

We also define the following block matrices

$$
\begin{aligned}
\left(A_{11}\right)_{i j} & =\left(\partial_{y}^{2} \tilde{\phi}_{j}, \tilde{\phi}_{i}\right)_{I_{1}}+k^{2}\left(\tilde{\phi}_{j}, \tilde{\phi}_{i}\right)_{I_{1}}, \\
\left(C_{11}\right)_{i j} & =\left(\partial_{y}^{2} \tilde{\phi}_{N_{y}-1+j}, \tilde{\phi}_{N_{y}-1+i}\right)_{I_{1}}+k^{2}\left(\tilde{\phi}_{N_{y}-1+j}, \tilde{\phi}_{N_{y}-1+i}\right)_{I_{2}}, \\
\left(B_{11}\right)_{i j} & =\left(\partial_{y}^{2} \tilde{\phi}_{2 N_{y}-2+j}, \tilde{\phi}_{2 N_{y}-2+i}\right)_{I_{3}}+k^{2}\left(\tilde{\phi}_{2 N_{y}-2+j}, \tilde{\phi}_{2 N_{y}-2+i}\right)_{I_{3}},
\end{aligned}
$$

where $0 \leq i, j \leq N_{y}-2$. We make the column vectors

$$
\begin{aligned}
& a_{12}=\left(\partial_{y}^{2} \tilde{\phi}_{3 N_{y}-3}+k^{2} \tilde{\phi}_{3 N_{y}-3}, \tilde{\phi}_{j}\right)_{I_{1}}, \\
& c_{12}=\left(\partial_{y}^{2} \tilde{\phi}_{3 N_{y}-3}+k^{2} \tilde{\phi}_{3 N_{y}-3}, \tilde{\phi}_{N_{y}-1+j}\right)_{I_{2}}, \\
& d_{12}=\left(\partial_{y}^{2} \tilde{\phi}_{3 N_{y}-2}+k^{2} \tilde{\phi}_{3 N_{y}-2}, \tilde{\phi}_{N_{y}-1+j}\right)_{I_{2}}, \\
& b_{12}=\left(\partial_{y}^{2} \tilde{\phi}_{3 N_{y}-2}+k^{2} \tilde{\phi}_{3 N_{y}-2}, \tilde{\phi}_{2 N_{y}-2+j}\right)_{I_{3}},
\end{aligned}
$$


and row vectors

$$
\begin{aligned}
& a_{21}=\left(\partial_{y}^{2} \tilde{\phi}_{j}+k^{2} \tilde{\phi}_{j}, \tilde{\phi}_{3 N_{y}-3}\right)_{I_{1}}+\partial_{y} \tilde{\phi}_{j}(\bar{g}), \\
& c_{21}=\left(\partial_{y}^{2} \tilde{\phi}_{N_{y}-1+j}+k^{2} \tilde{\phi}_{N_{y}-1+j}, \tilde{\phi}_{3 N_{y}-3}\right)_{I_{2}}-\tau^{2} \partial_{y} \tilde{\phi}_{N_{y}-1+j}(\bar{g}), \\
& d_{21}=\left(\partial_{y}^{2} \tilde{\phi}_{N_{y}-1+j}+k^{2} \tilde{\phi}_{N_{y}-1+j}, \tilde{\phi}_{3 N_{y}-2}\right)_{I_{2}}+\sigma^{-2} \partial_{y} \tilde{\phi}_{N_{y}-1+j}(\bar{h}), \\
& b_{21}=\left(\partial_{y}^{2} \tilde{\phi}_{2 N_{y}-2+j}+k^{2} \tilde{\phi}_{2 N_{y}-2+j}, \tilde{\phi}_{3 N_{y}-2}\right)_{I_{3}}-\partial_{y} \tilde{\phi}_{2 N_{y}-2+j}(\bar{h}),
\end{aligned}
$$

for $0 \leq j \leq N_{y}-2$. Finally, we set

$$
\begin{aligned}
a_{22}= & \left(\partial_{y}^{2} \tilde{\phi}_{3 N_{y}-3}+k^{2} \tilde{\phi}_{3 N_{y}-3}, \tilde{\phi}_{3 N_{y}-3}\right)+\partial_{y} \tilde{\phi}_{3 N_{y}-3}\left(\bar{g}^{+}\right)-\tau^{2} \partial_{y} \tilde{\phi}_{3 N_{y}-3}\left(\bar{g}^{-}\right), \\
a_{33}= & \left(\partial_{y}^{2} \tilde{\phi}_{3 N_{y}-2}+k^{2} \tilde{\phi}_{3 N_{y}-2}, \tilde{\phi}_{3 N_{y}-2}\right)+\sigma^{-2} \partial_{y} \tilde{\phi}_{3 N_{y}-2}\left(\bar{h}^{+}\right)-\partial_{y} \tilde{\phi}_{3 N_{y}-2}\left(\bar{h}^{-}\right), \\
a_{23}= & \left(\partial_{y}^{2} \tilde{\phi}_{3 N_{y}-2}+k^{2} \tilde{\phi}_{3 N_{y}-2}, \tilde{\phi}_{3 N_{y}-3}\right) \\
& +\left[\partial_{y} \tilde{\phi}_{3 N_{y}-2}\left(\bar{g}^{+}\right)-\tau^{2} \partial_{y} \tilde{\phi}_{3 N_{y}-2}\left(\bar{g}^{-}\right)\right] \tilde{\phi}_{3 N_{y}-3}(\bar{g}), \\
a_{32}= & \left(\partial_{y}^{2} \tilde{\phi}_{3 N_{y}-3}+k^{2} \tilde{\phi}_{3 N_{y}-3}, \tilde{\phi}_{3 N_{y}-2}\right) \\
& +\left[\sigma^{-2} \partial_{y} \tilde{\phi}_{3 N_{y}-3}\left(\bar{h}^{+}\right)-\partial_{y} \tilde{\phi}_{3 N_{y}-3}\left(\bar{h}^{-}\right)\right] \tilde{\phi}_{3 N_{y}-2}(\bar{h}) .
\end{aligned}
$$

With these our Legendre-Galerkin scheme becomes the following system of $3 N_{y}-1$ equations:

$$
\left(\begin{array}{ccccc}
A_{11} & 0 & 0 & a_{12} & 0 \\
0 & C_{11} & 0 & c_{12} & d_{12} \\
0 & 0 & B_{11} & 0 & b_{12} \\
a_{21}^{T} & c_{21}^{T} & 0 & a_{22} & a_{23} \\
0 & d_{21}^{T} & b_{21}^{T} & a_{32} & a_{33}
\end{array}\right)\left(\begin{array}{c}
\mathbf{u} \\
\mathbf{v} \\
\mathbf{w} \\
\hat{u}_{3 N_{y}-3} \\
\hat{u}_{3 N_{y}-2}
\end{array}\right)=\left(\begin{array}{c}
\mathbf{f} \\
\\
\hat{f}_{3 N_{y}-3} \\
\hat{f}_{3 N_{y}-2}
\end{array}\right) .
$$

\subsection{Simulations of a Simplified Model}

To begin our numerical experiments we considered the approximation of the "reduced" boundary value problem (7) at the heart of our full solver. 
With this we displayed the convergence performance we anticipated for the full algorithm proposed in Section 5. For this we considered the following problem.

$$
\begin{array}{ll}
\partial_{y}^{2} u(y)+k_{u}^{2} u(y)=\mathcal{F}_{1}(y), & \bar{g}<y<a \\
\partial_{y}^{2} v(y)+k_{v}^{2} v(y)=\mathcal{F}_{2}(y), & \bar{h}<y<\bar{g} \\
\partial_{y}^{2} w(y)+k_{w}^{2} w(y)=\mathcal{F}_{3}(y), & b<y<\bar{h} \\
u(\bar{g})-v(\bar{g})=0, & \\
v(\bar{h})-w(\bar{h})=0, & \\
\partial_{y} u(\bar{g})-\tau^{2} \partial_{y} v(\bar{g})=0, & \\
\partial_{y} v(\bar{h})-\sigma^{2} \partial_{y} w(\bar{h})=0, & y=a, \\
\partial_{y} u-\mathrm{i} \beta^{u} u=0, & y=b . \\
\partial_{y} w+\mathrm{i} \beta^{w} w=0, &
\end{array}
$$

To test the convergence of our algorithm we began by considering the following functions and parameters which we used to define an exact solution:

$$
\begin{gathered}
u=\exp (y)(y-a)^{2}(y-b)^{2}, \quad v=\exp (y)(y-a)^{2}(y-b)^{2}, \\
w=\exp (y)(y-a)^{2}(y-b)^{2}, \\
a=2.5, \quad \bar{g}=1, \quad \bar{h}=0, \quad b=-2, \quad \tau=1, \quad \sigma=1, \\
k_{u}=5.0, \quad k_{v}=1.0, \quad k_{w}=1.1 \\
\beta_{u}=-2+2 \mathrm{i}, \quad \beta_{w}=1-\mathrm{i} .
\end{gathered}
$$

One can check that the functions $u, v$, and $w$ satisfy the homogeneous boundary conditions in (8). One can also easily find the corresponding terms $\mathcal{F}_{1}, \mathcal{F}_{2}$, and $\mathcal{F}_{3}$ which make $u, v$, and $w$ exact solutions.

As a second example, we considered $\tau \neq 1$ and $\sigma \neq 1$, which was meant to model a TM simulation:

$$
\begin{gathered}
u=\tau^{2} \sigma^{2} \exp (y)(y-a)^{2}(y-b)^{2}(y-\bar{g})(y-\bar{h}), \\
v=\sigma^{2} \exp (y)(y-a)^{2}(y-b)^{2}(y-\bar{g})(y-\bar{h}), \\
w=\exp (y)(y-a)^{2}(y-b)^{2}(y-\bar{g})(y-\bar{h}), \\
a=5, \quad \bar{g}=3, \quad \bar{h}=0, \quad b=-2, \quad \tau=2, \quad \sigma=4, \\
k_{u}=3.0, \quad k_{v}=1+\mathrm{i}, \quad k_{w}=2.0, \\
\beta_{u}=2+\mathrm{i}, \quad \beta_{w}=1-\mathrm{i} .
\end{gathered}
$$


135

To test numerical convergence, we defined the relative $L^{2}$ error

$$
\frac{\left\|u_{\mathrm{ex}}-u_{N_{y}}\right\|_{L^{2}}}{\left\|u_{\mathrm{ex}}\right\|_{L^{2}}}
$$

136 solution of (8). Figures 3 and 4 display the spectral rate of convergence

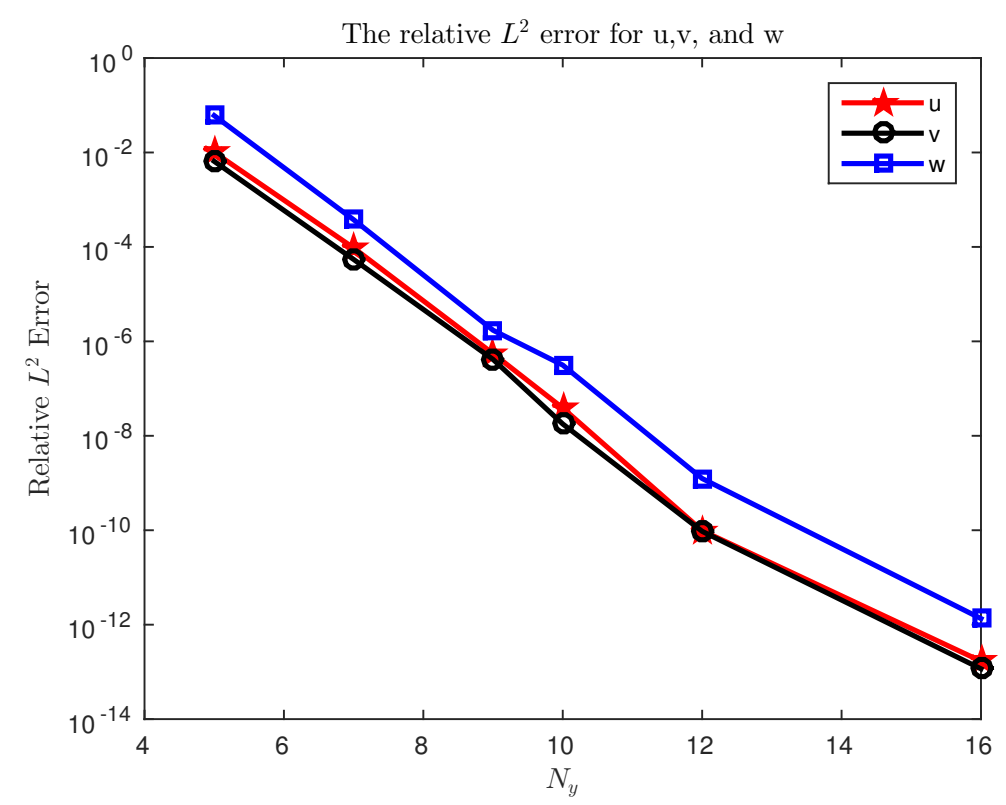

Figure 3: Relative $L^{2}$ error, (11), of our Legendre-Galerkin approximation of (8) with parameter choices (9) versus number of basis functions $N_{y}$.

which our Legendre-Galerkin method achieved in this simplified setting.

\subsection{Simulations of a Triply Layered Medium: Smooth Interfaces}

We now display results of simulations of a triply layered medium whose scattering returns are governed by (2). As exact solutions are unavailable, to display the spectral accuracy of our Legendre-Galerkin scheme we use the widely-accepted diagnostic of "energy defect"; see e.g. [65, 16, 39, 40, 41].

To describe this we consider the Rayleigh expansions [37] in the upper and lower layers

$$
u(x, y)=\sum_{p=-\infty}^{\infty} \hat{u}_{p} e^{\mathrm{i} \alpha_{p} x+\mathrm{i} \beta_{p}^{u} y}, \quad w(x, y)=\sum_{p=-\infty}^{\infty} \hat{w}_{p} e^{\mathrm{i} \alpha_{p} x-\mathrm{i} \beta_{p}^{w} y}
$$




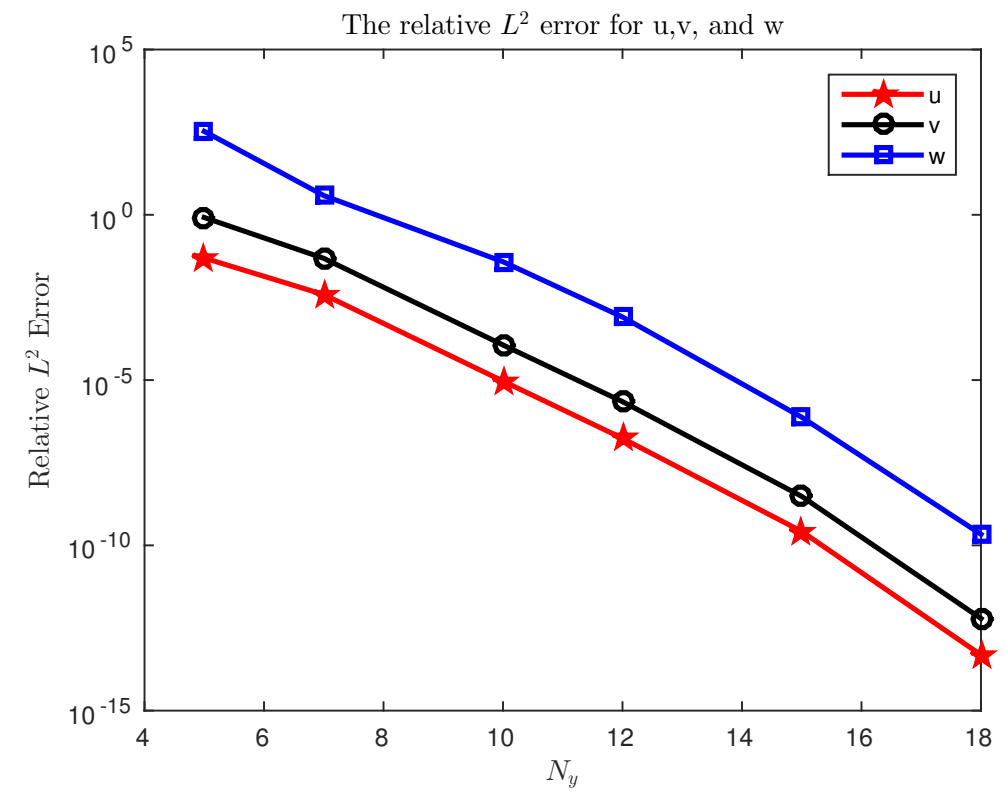

Figure 4: Relative $L^{2}$ error, (11), of our Legendre-Galerkin approximation of (8) with parameter choices (10) versus number of basis functions $N_{y}$.

and, from these, define the efficiencies

$$
\begin{aligned}
e_{p}^{u}:=\frac{\beta_{p}^{u}}{\beta^{u}}\left|\hat{u}_{p}\right|^{2}, & p \in \mathcal{U}^{u} \\
e_{p}^{w}:=\frac{\beta_{p}^{w}}{\beta^{u}}\left|\hat{w}_{p}\right|^{2}, & p \in \mathcal{U}^{w}
\end{aligned}
$$

The efficiencies measure the energy at wavenumber $p$ propagated away from the grating interface, and if all materials in the structure are lossless $\left(n^{m} \in\right.$ $\mathbf{R}$ ), we obtain conservation of energy. More precisely, in TE mode

$$
\sum_{p \in \mathcal{U}^{u}} e_{p}^{u}+\sum_{p \in \mathcal{U}^{w}} e_{p}^{w}=1
$$

while in TM mode

$$
\sum_{p \in \mathcal{U}^{u}} e_{p}^{u}+\left(\frac{k_{u}^{2}}{k_{w}^{2}}\right) \sum_{p \in \mathcal{U}^{w}} e_{p}^{w}=1
$$


In particular, we can define the "energy defect" [65] for TE polarization as

$$
\delta_{\mathrm{TE}}:=1-\sum_{p \in \mathcal{U}^{u}} e_{p}^{u}-\sum_{p \in \mathcal{U}^{w}} e_{p}^{w}
$$

and for TM polarization as

$$
\delta_{\mathrm{TM}}:=1-\sum_{p \in \mathcal{U}^{u}} e_{p}^{u}-\left(\frac{k_{u}^{2}}{k_{w}^{2}}\right) \sum_{p \in \mathcal{U}^{w}} e_{p}^{w},
$$

which should be zero for an exact solution.

We conducted a sequence of simulations to show the spectral accuracy of our proposed Legendre-Galerkin algorithm. For a series of smooth interface tests, we used the following parameters

$$
\begin{gathered}
a=5, \quad \bar{g}=2, \quad \bar{h}=1, \quad b=-2, \quad \alpha=0.1, \\
d=2 \pi, \quad g(x)=\sin (x), \quad h(x)=\sin (x / 2) .
\end{gathered}
$$

For numerical parameters we used $N$ to denote the perturbation order, $N_{x}$ the number of Fourier modes in $x$, and $N_{y}$ the number of Galerkin coefficients in $y$. Finally, $\varepsilon$ was the boundary deformation height/slope, while $k_{u}, k_{v}$, and $k_{w}$ were the wavenumbers in the upper, middle, and lower layers, respectively.

In Figures 5 and 6 we display the energy defect versus number of perturbation orders retained for the parameter choices $N_{x}=N_{y}=24$ and $\left(k_{u}, k_{v}, k_{w}\right)=(1.5,2.5,3.5)$. We show this for TE and TM modes with various values of $\varepsilon$. The figures show the spectral convergence of the energy defect as the perturbation order $N$ is refined. We also found that the energy defect decays more quickly to machine precision for smaller choices of $\varepsilon$.

We repeated this experiment for fixed value of $N$ while varying the spatial discretization parameters $N_{x}$ and $N_{y}$. The results are displayed in Figures 7 and 8 for $N=20$ and various values of $N_{x}$ and $N_{y}$. Once again, we chose $\left(k_{u}, k_{v}, k_{w}\right)=(1.5,2.5,3.5)$ and performed simulations for TE and TM modes for a selection of $\varepsilon$. Once again we saw the spectral convergence of the energy defect, this time versus the number of Fourier and Legendre coefficients.

We then focused on the behavior of our method as $\varepsilon$ was increased. For this we fixed $N_{x}=22$ and $N_{y}=24$, and once again choose $\left(k_{u}, k_{v}, k_{w}\right)=$ $(1.5,2.5,3.5)$. We conducted numerical experiments in both TE and TM polarizations, and display the results in Figures 9 and 10. These figures depict the energy defect versus the perturbation size $\varepsilon$ with various perturbation 


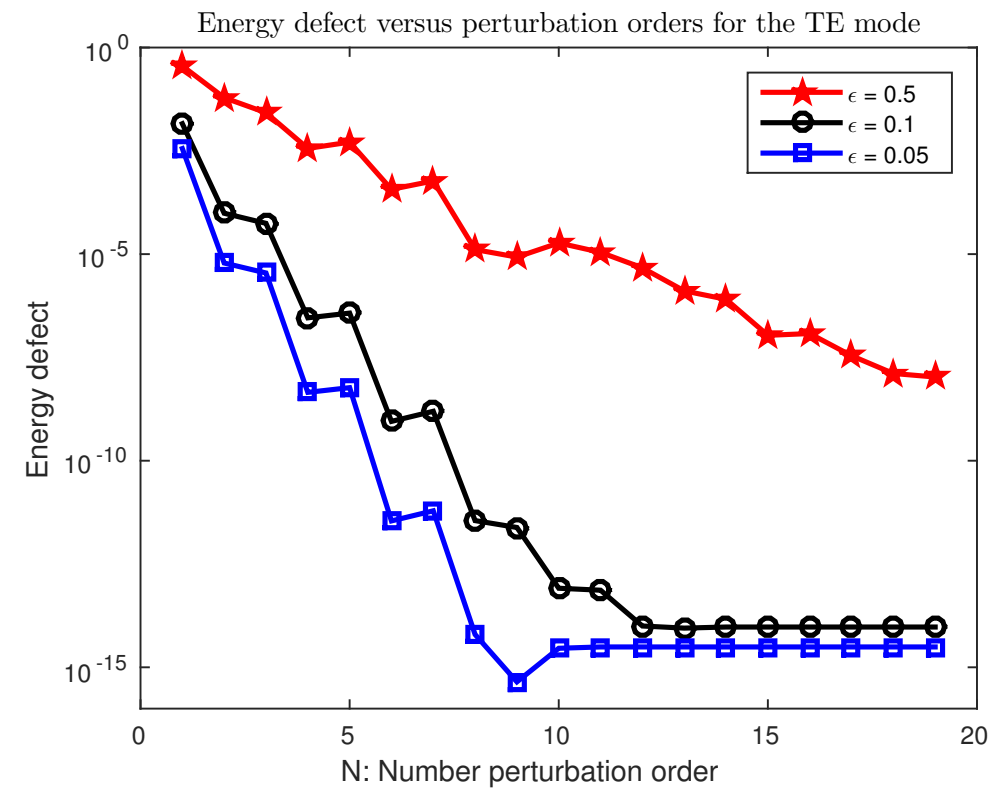

Figure 5: Energy defect versus perturbation order, $N$, for smooth interface configuration (12): TE mode.

orders $N$. As expected, smaller errors are realized for smaller choices of $\varepsilon$ and larger values of $N$.

Finally, in Figures 11 and 12 we computed the energy defect for fixed $\varepsilon=0.01, N=15, N_{x}=20$, and $N_{y}=40$ (for TE and TE modes) with various choices of wavenumber. If the collection of wavenumbers was not large (e.g. $\left.\left(k_{u}, k_{v}, k_{w}\right)=(1.5,2.5,3.5)\right)$, then we observed rapid convergence to machine precision. However, as we increased to larger values of $k_{m}$ our convergence deteriorated due to the severe under-resolution of our parameter choices. To address this latter concern we revisited these computations and found that if we refined our spatial discretization then we could recover the striking convergence we saw for the smallest values of $\left(k_{u}, k_{v}, k_{w}\right)$. To be more specific, as we display in Figure 13, if we chose $N_{x}=30$ and $N_{y}=60$ we realized accuracies of $10^{-14}$ to $10^{-15}$ for the moderate values $\left(k_{u}, k_{v}, k_{w}\right)=$ $(11.5,12.5,13.5)$. Furthermore, if we chose $N_{x}=40$ and $N_{y}=100$ then we realized errors on the order of $10^{-14}$ for the large values $\left(k_{u}, k_{v}, k_{w}\right)=$ $(41.5,42.5,43.5)$. 


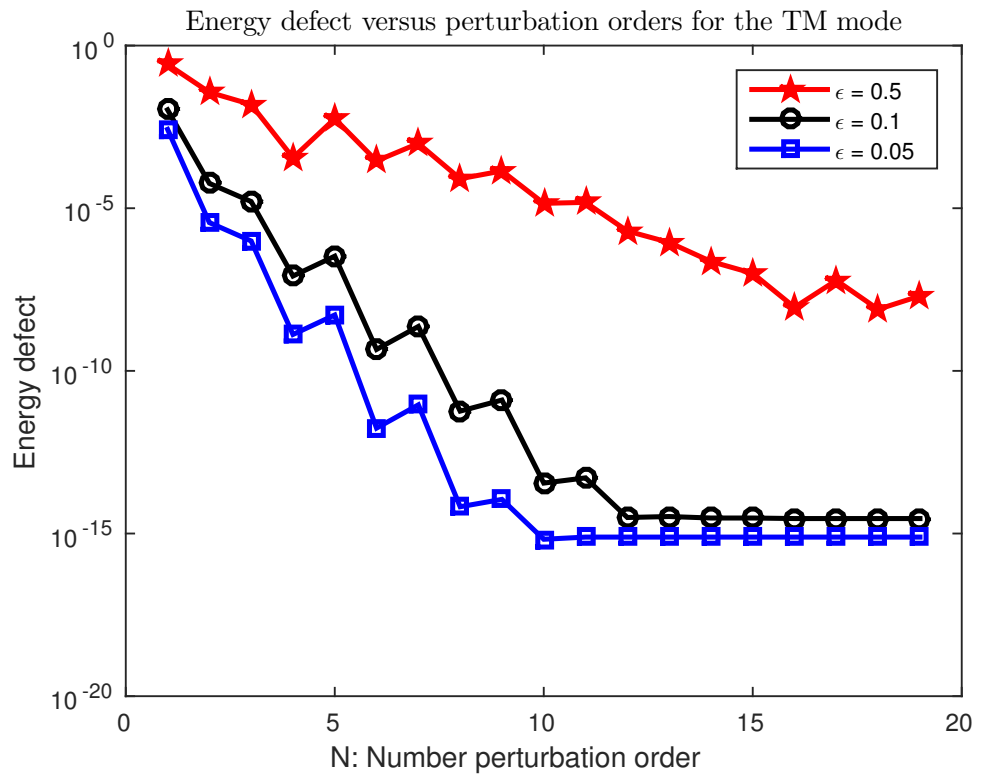

Figure 6: Energy defect versus perturbation order, $N$, for smooth interface configuration (12): TM mode.

\subsection{Simulations of a Triply Layered Medium: Rough Interfaces}

We repeated the first simulation of the previous section specified in (12) with the smooth profiles replaced by the much rougher profiles

$$
\begin{aligned}
& f_{r}(x)=\left(2 \times 10^{-4}\right) x^{4}(2 \pi-x)^{4}-c_{0}, \\
& f_{L}(x)= \begin{cases}-\frac{2}{\pi} x+1, & 0 \leq x \leq \pi, \\
\frac{2}{\pi} x-3, & \pi \leq x \leq 2 \pi,\end{cases}
\end{aligned}
$$

(with $c_{0}$ chosen such that $f_{r}$ has zero mean) which possess $C^{4}$ (but not $C^{5}$ ) and Lipschitz regularity, respectively [48]. For our experiments we used their Fourier series representations

$$
\begin{aligned}
& f_{r}(x)=\sum_{k=1}^{\infty} \frac{96\left(2 k^{2} \pi^{2}-21\right)}{125 k^{8}} \cos (k x) \\
& f_{L}(x)=\sum_{k=1}^{\infty} \frac{8}{\pi^{2}(2 k-1)^{2}} \cos ((2 k-1) x),
\end{aligned}
$$




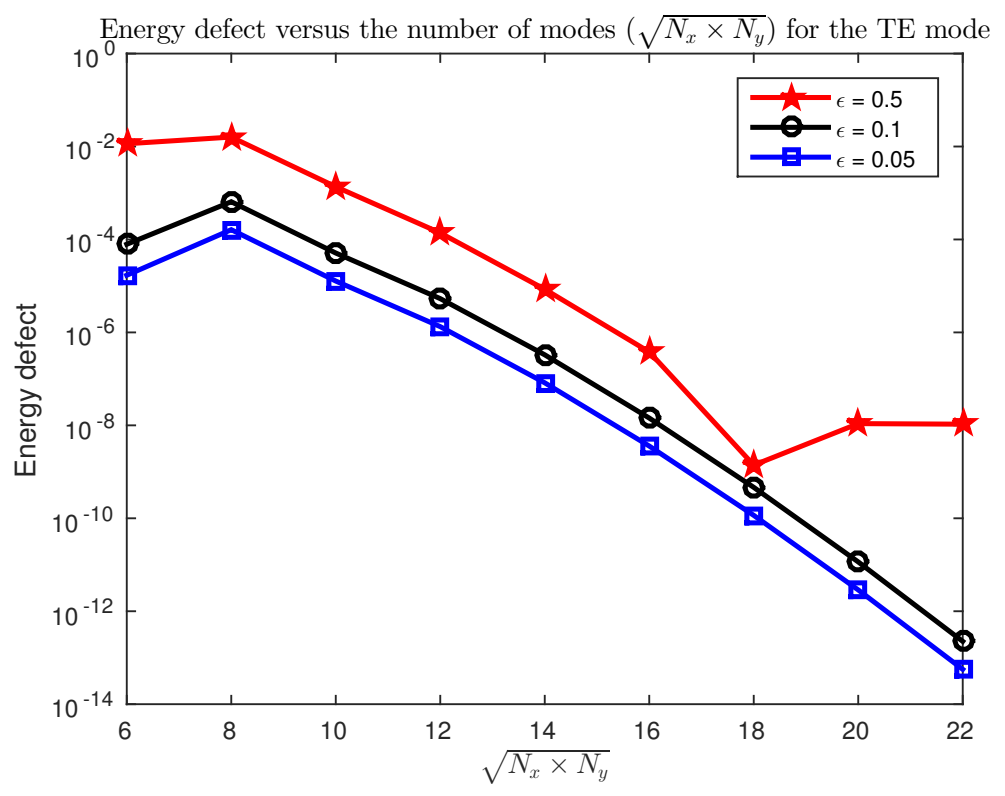

Figure 7: Energy defect versus number of modes, $\sqrt{N_{x} N_{y}}$, for smooth interface configuration (12): TE mode.

which we truncated after wavenumber $P=40$,

$$
\begin{aligned}
& f_{r, P}(x)=\sum_{k=1}^{P} \frac{96\left(2 k^{2} \pi^{2}-21\right)}{125 k^{8}} \cos (k x) \\
& f_{L, P}(x)=\sum_{k=1}^{P / 2} \frac{8}{\pi^{2}(2 k-1)^{2}} \cos ((2 k-1) x) .
\end{aligned}
$$

The specific details of our numerical simulations are given by the following parameters.

$$
\begin{gathered}
a=5, \quad \bar{g}=2, \quad \bar{h}=1, \quad b=-2, \quad \alpha=0.1, \\
d=2 \pi, \quad g(x)=f_{r, 40}(x) \quad h(x)=f_{L, 40}(x) .
\end{gathered}
$$

In addition, we picked $\left(k_{u}, k_{v}, k_{w}\right)=(1.5,2.5,3.5), \varepsilon=0.05, N=15$, and three choices of $\left(N_{x}, N_{y}\right)$. In Figure 14 we display the results of these experiments and show that, provided with sufficient resolution, our new approach 


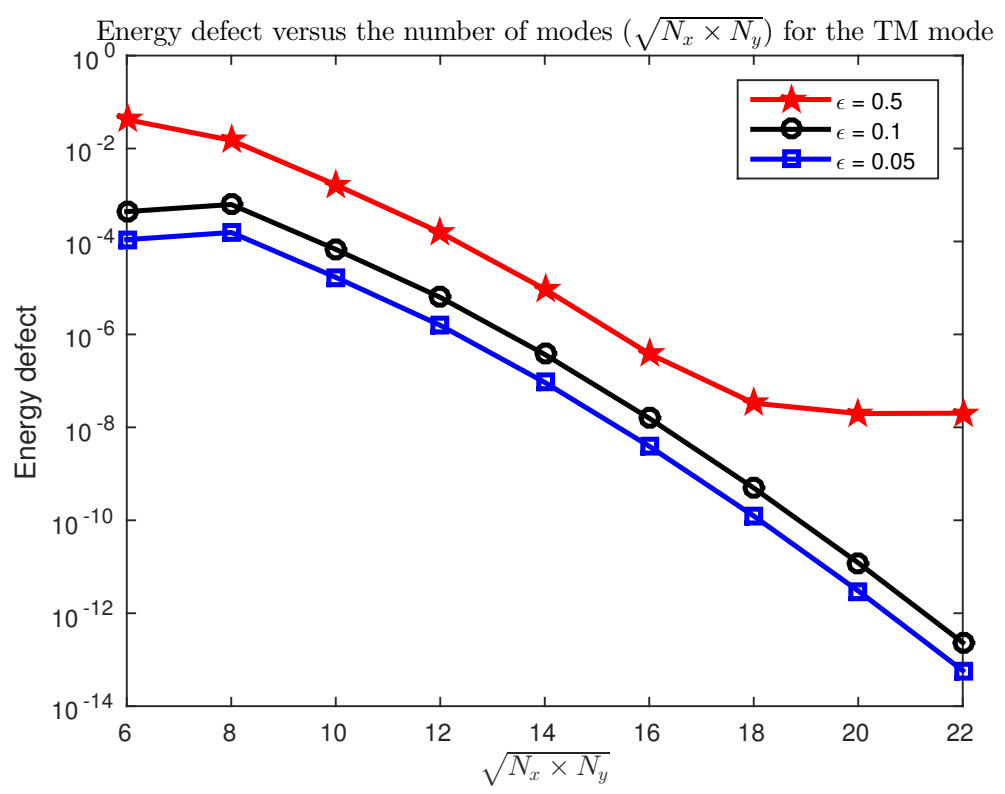

Figure 8: Energy defect versus number of modes, $\sqrt{N_{x} N_{y}}$, for smooth interface configuration (12): TM mode.

is equally applicable to configurations with finite and even Lipschitz smoothness. In Figures 15 and 16 we show the real and imaginary parts of the scattered solution. The layer interfaces are depicted in dashed red lines.

To further investigate the capabilities of our algorithm, we revisit this computation with different values of $P$, more precisely $P=20,40,80,160$. The results of this study are summarized in Figure 17 which show that, while the accuracy deteriorates a small amount as $P$ increases (representing a more faithful representation of the very rough interfaces), it remains quite small throughout the entire range of $P$ and shows no sign of producing divergent results regardless of how large $P$ is chosen.

To close our study we reconsider the smooth profile configuration, (12), but replace the choice of $k_{v}$ with 42.5 meant to simulate a device with "highcontrast media." In Figure 18 we report the results of this experiment and see, once again, that given sufficient resolution we can realize results with excellent accuracy. 


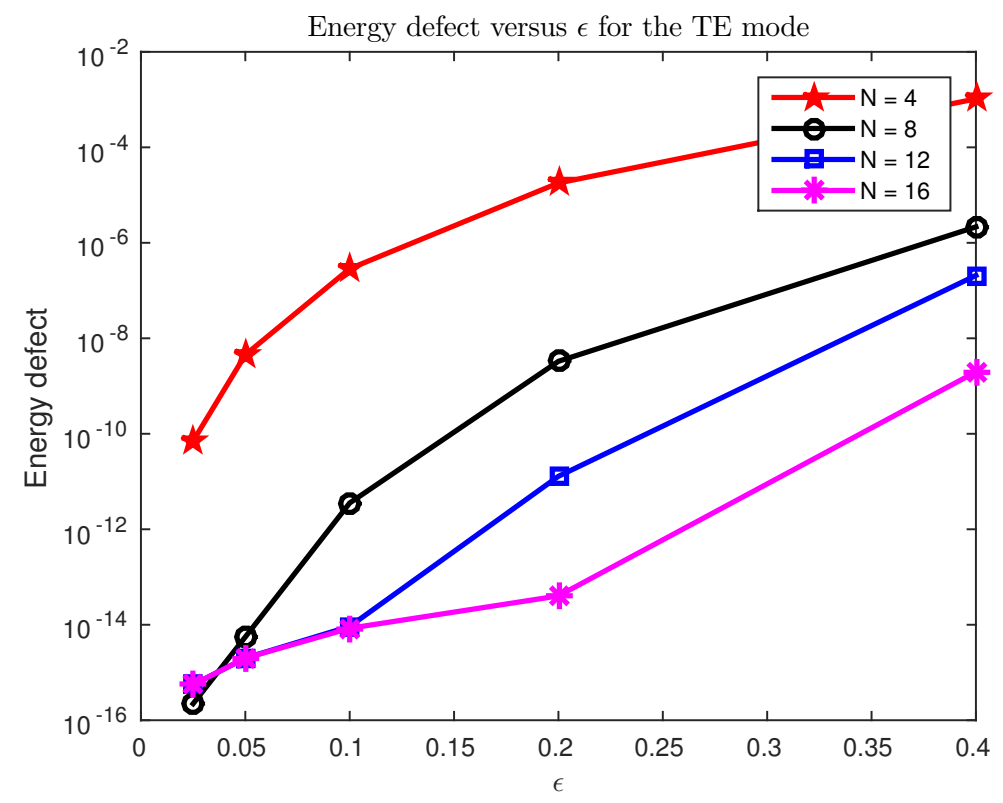

Figure 9: Energy defect versus perturbation size, $\varepsilon$, for smooth interface configuration (12): TE mode.

\section{Conclusions}

We have described a High-Order Perturbation of Surfaces (HOPS) algorithm for electromagnetic scattering in a triply layered periodic medium. We equivalently restated the governing Helmholtz equations for this layered medium on a bounded domain using Dirichlet-Neumann operators at artificial boundaries above and below the structure. Utilizing a suitable change of variables, we were able to study the governing equations on a separable geometry with flat interfaces. Introducing boundary perturbations, we expressed the scattered field in a Taylor series and derived a sequence of linear problems to be solved at each perturbation order for the $n$-th order term in this expansion, resulting in a Transformed Field Expansions (TFE) algorithm. Accurate numerical simulations of these TFE recursions were performed with a Legendre-Galerkin method based on a novel weak formulation. Our numerical simulations show the spectral convergence which our new algorithm can achieve.

Our developments clearly point towards several extensions of great im- 


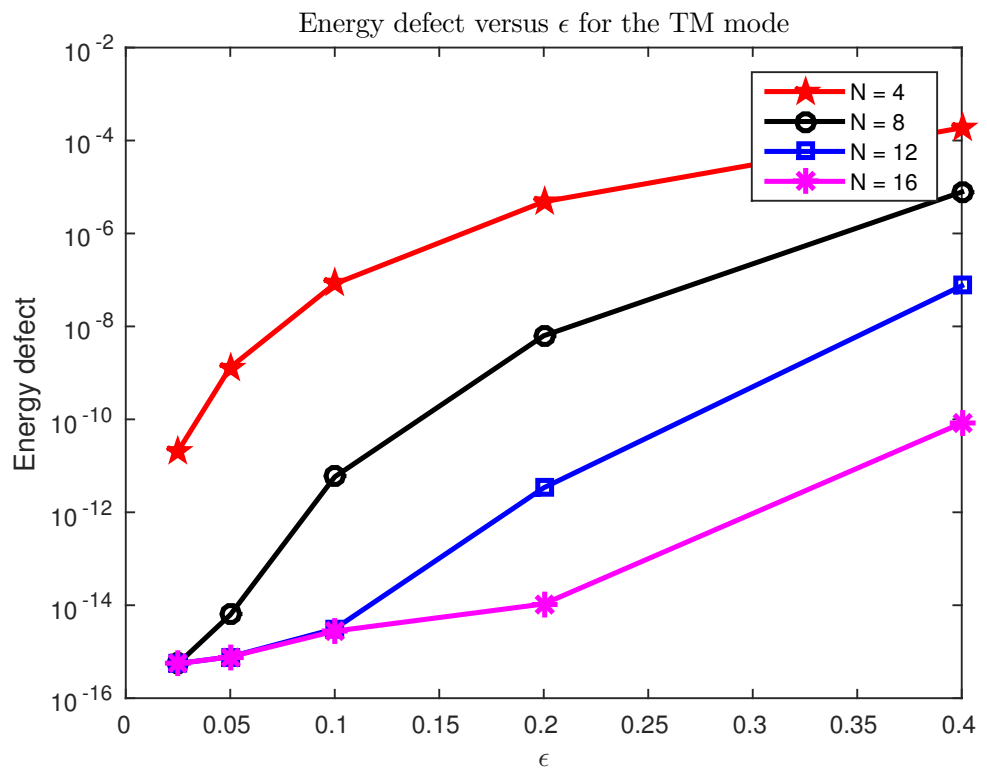

Figure 10: Energy defect versus perturbation size, $\varepsilon$, for smooth interface configuration (12): TM mode.

portance. In particular, our approach must be generalized to an arbitrary number of layers. While the extension is, in some sense, straightforward given the developments of this contribution, the algorithmic differences are significant and we will describe them in a future publication. In addition, the current approach, which models the scattering of scalar waves relevant to acoustics, must be extended to the vector wave scattering problems of electromagnetics (in three dimensions) and linear elastodynamics.

\section{Acknowledgements}

Y.H. gratefully acknowledges support from the Simons Foundation. D.P.N. gratefully acknowledges support from the National Science Foundation through grant No. DMS-1522548.

\section{Appendix A. Derivation of the Transformed Equations}




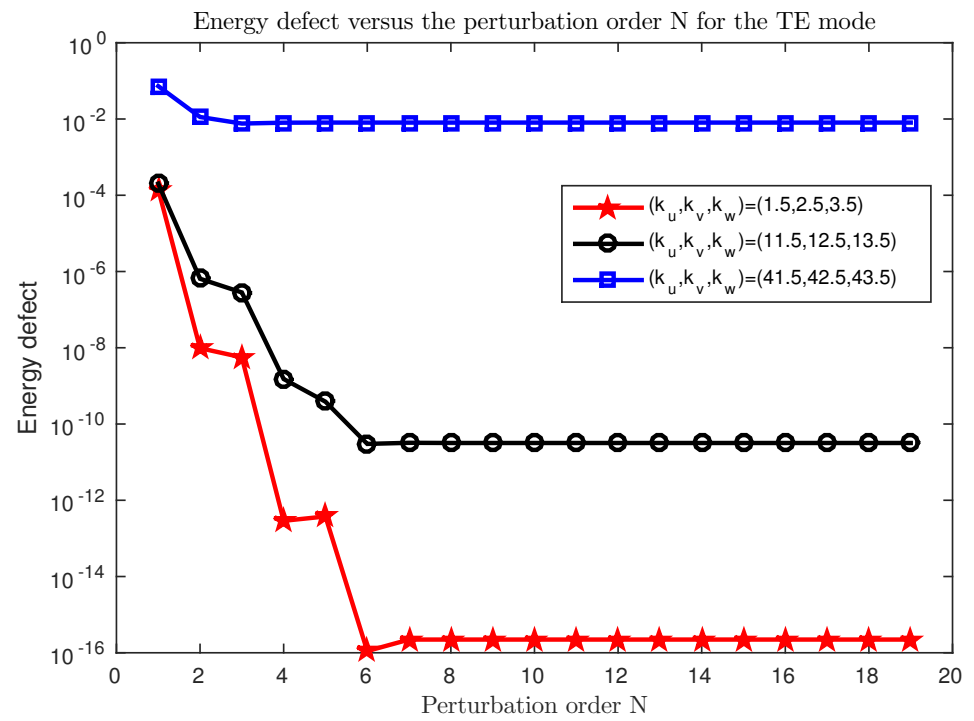

Figure 11: Energy defect versus perturbation order, $N$, for smooth interface configuration (12): TE mode.

In this section we give a full derivation of the transformed version of the 231 governing equations (2) presented in Section 3.1.

Remark 1. As our problem geometry features three distinct layers, two of which are similar (the upper and lower) and one of which (the middle) has quite different character, the notation can become cumbersome. In order to simplify and shorten our presentation we give formulas for the upper and lower layers together, while separating the middle layer formulas from these. To achieve this we assign the upper and lower layers indices $i=1$ and $i=3$, respectively, and define the middle layer by $i=2$. In addition we use the following notation for the geometrical aspects of each domain: In the upper layer

$$
u_{1}:=u, \quad \xi_{1}:=a, \quad \bar{\varphi}_{1}:=\bar{g}, \quad \varphi_{1}(x):=g(x), \quad g(x)=\varepsilon \psi_{1}(x),
$$

the latter in anticipation of our boundary perturbation methodology. In the lower domain we define

$$
u_{3}:=w, \quad \xi_{3}:=b, \quad \bar{\varphi}_{3}:=\bar{h}, \quad \varphi_{3}(x):=h(x), \quad h(x)=\varepsilon \psi_{3}(x) .
$$




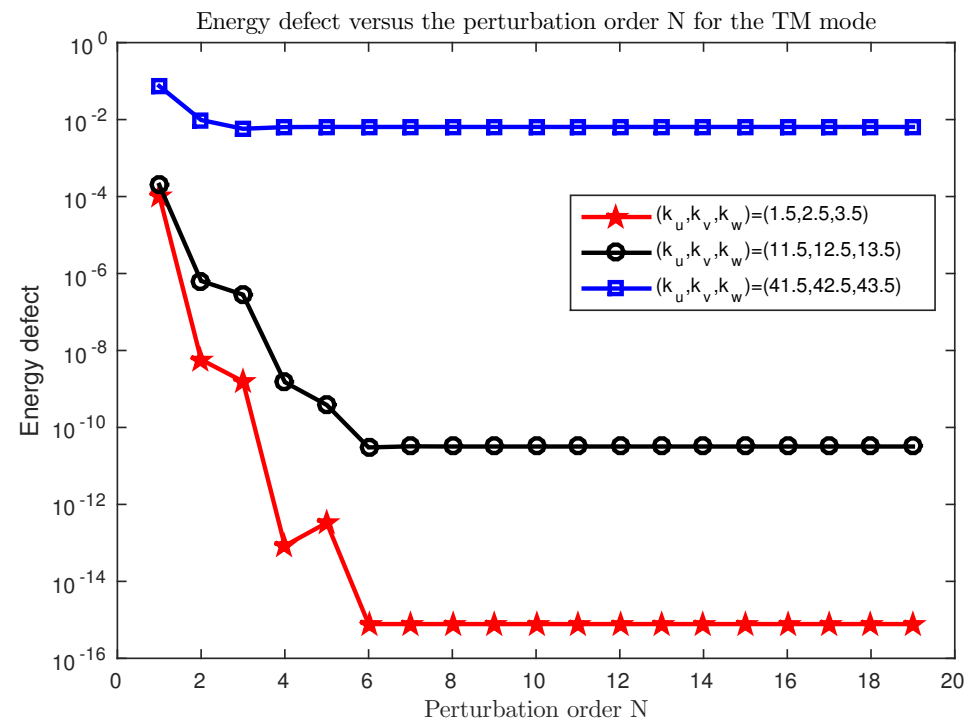

Figure 12: Energy defect versus perturbation order, $N$, for smooth interface configuration (12): TM mode.

In the middle layer $(i=2)$ we find it more useful to simply define

$$
u_{2}:=v, \quad g(x)=\varepsilon f_{g}(x), \quad h(x)=\varepsilon f_{h}(x),
$$

where we note that $\left\{f_{g}, f_{h}\right\}$ are just different names for $\left\{\psi_{1}, \psi_{3}\right\}$ defined above. In these, $\left\{\psi_{1}=f_{g}, \psi_{3}=f_{h}\right\}$ are the order one boundary perturbation shapes.

Returning to our change of variables, by the chain rule we find

$$
\begin{aligned}
& \partial_{x}=\partial_{x^{\prime}}+\left(\partial_{x} y_{i}\right) \partial_{y_{i}} \\
& \partial_{y}=\left(\partial_{y} y_{i}\right) \partial_{y_{i}},
\end{aligned}
$$

for $i=1,2,3$. For $i=1,3$, we have

$$
\left(x^{\prime}, y_{i}\right)=\left(x, \xi_{i}\left(\frac{y-\left(\bar{\varphi}_{i}+\varphi_{i}\right)}{\xi_{i}-\left(\bar{\varphi}_{i}+\varphi_{i}\right)}\right)+\bar{\varphi}_{i}\left(\frac{\xi_{i}-y}{\xi_{i}-\left(\bar{\varphi}_{i}+\varphi_{i}\right)}\right)\right),
$$




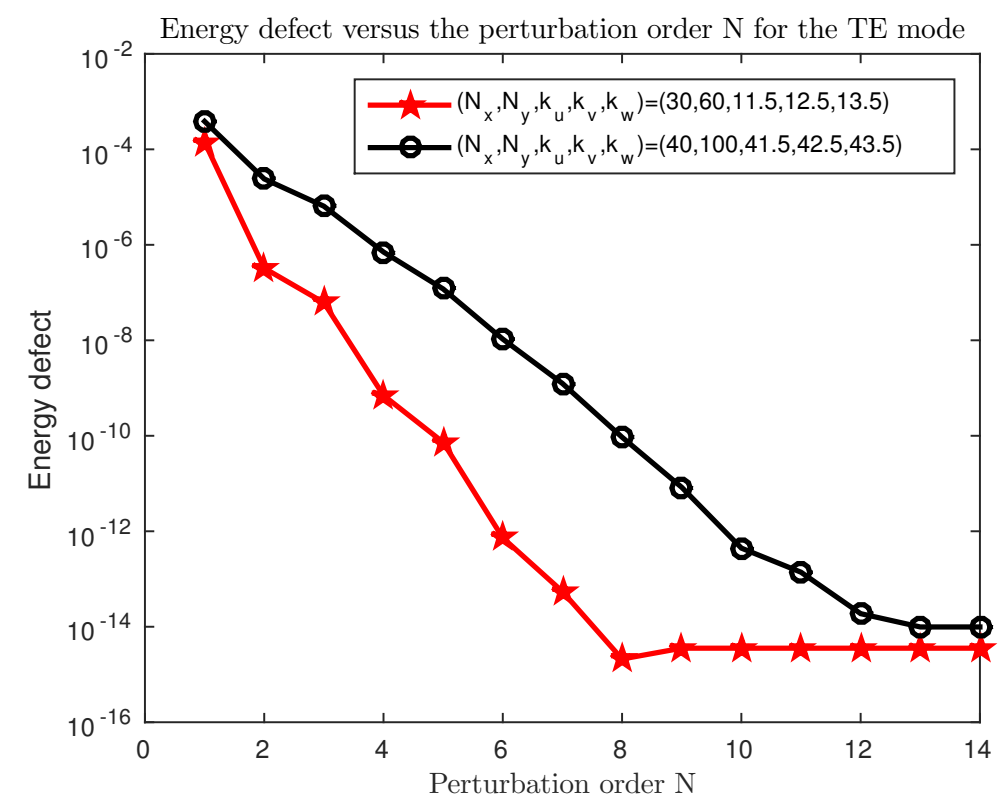

Figure 13: Energy defect versus perturbation order, $N$, for two higher wavenumber combinations for smooth interface configuration (12): TE mode.

and noting that

$$
\begin{aligned}
{\left[\xi_{i}-\left(\bar{\varphi}_{i}+\varphi_{i}\right)\right] \partial_{x}=} & {\left[\xi_{i}-\left(\bar{\varphi}_{i}+\varphi_{i}\right)\right] \partial_{x^{\prime}}+\left[\xi_{i}-\left(\bar{\varphi}_{i}+\varphi_{i}\right)\right] \partial_{x} y_{i} \partial_{y_{i}} } \\
= & {\left[\xi_{i}-\left(\bar{\varphi}_{i}+\varphi_{i}\right)\right] \partial_{x^{\prime}}+\partial_{x}\left(\left[\xi_{i}-\left(\bar{\varphi}_{i}+\varphi_{i}\right)\right] y_{i}\right) \partial_{y_{i}} } \\
& -y_{i} \partial_{x}\left[\xi_{i}-\left(\bar{\varphi}_{i}+\varphi_{i}\right)\right] \partial_{y_{i}}
\end{aligned}
$$

we discover that

$$
\begin{aligned}
& {\left[\xi_{i}-\left(\bar{\varphi}_{i}+\varphi_{i}\right)\right] \partial_{x}=\left[\xi_{i}-\left(\bar{\varphi}_{i}+\varphi_{i}\right)\right] \partial_{x^{\prime}}+\partial_{x^{\prime}} \varphi_{i}\left(y_{i}-\xi_{i}\right) \partial_{y_{i}},} \\
& {\left[\xi_{i}-\left(\bar{\varphi}_{i}+\varphi_{i}\right)\right] \partial_{y}=\left(\xi_{i}-\bar{\varphi}_{i}\right) \partial_{y_{i}} .}
\end{aligned}
$$

On the other hand, for $i=2$,

$$
\left(x^{\prime}, y_{2}\right)=\left(x^{\prime}, \bar{h}\left(\frac{y-(\bar{g}+g)}{(\bar{h}+h)-(\bar{g}+g)}\right)+\bar{g}\left(\frac{(\bar{h}+h)-y}{(\bar{h}+h)-(\bar{g}+g)}\right)\right) .
$$




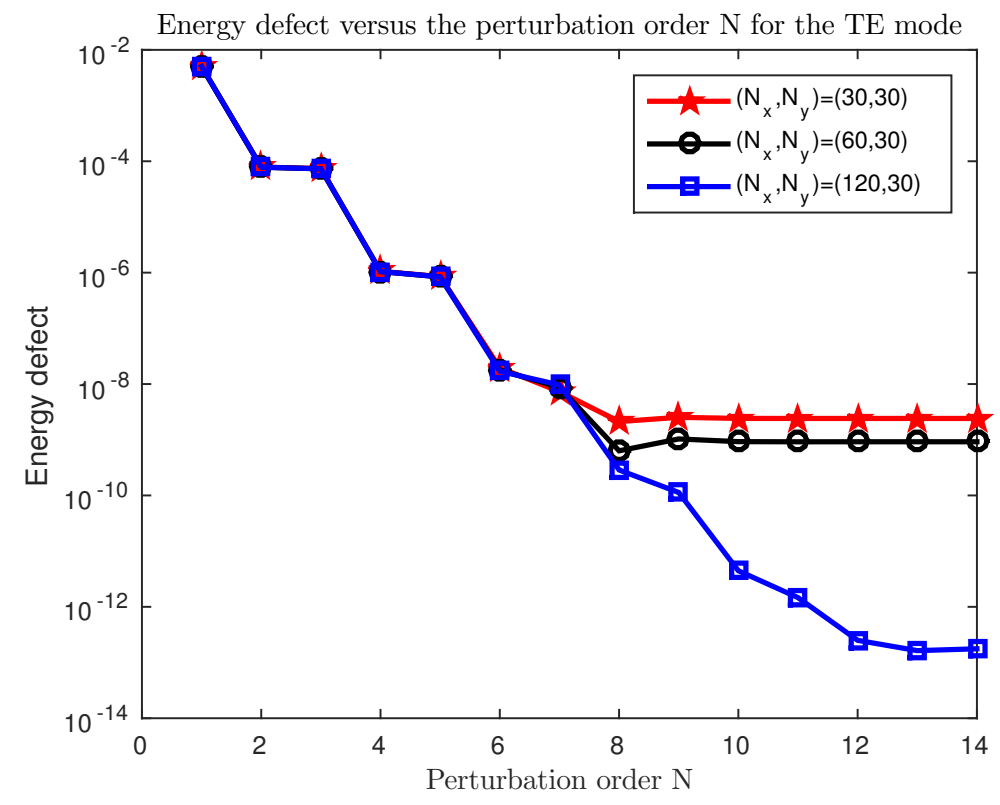

Figure 14: Energy defect versus perturbation order, $N$, for rough interface configuration (13): TE mode.

Setting $M(x)=(\bar{h}+h)-(\bar{g}+g)$, we deduce that

$$
\begin{aligned}
M \partial_{x} & =M \partial_{x^{\prime}}+M\left(\partial_{x} y_{2}\right) \partial_{y_{2}}=M \partial_{x^{\prime}}+\left[\partial_{x}\left(M y_{2}\right)-\left(\partial_{x} M\right) y_{2}\right] \partial_{y_{2}} \\
& =M \partial_{x^{\prime}}+\left[\bar{h}\left(-\partial_{x} g\right)+\bar{g} \partial_{x} h-\left(\partial_{x} h-\partial_{x} g\right) y_{2}\right] \partial_{y_{2}} \\
& =M \partial_{x^{\prime}}+\left[-\bar{h} \partial_{x} g+\bar{g} \partial_{x} h-\partial_{x} h y_{2}+\partial_{x} g y_{2}\right] \partial_{y_{2}} \\
& =M \partial_{x^{\prime}}+\left[\partial_{x} g\left(y_{2}-\bar{h}\right)-\partial_{x} h\left(y_{2}-\bar{g}\right)\right] \partial_{y_{2}} .
\end{aligned}
$$

Hence, we obtain that

$$
M \partial_{x}=M \partial_{x^{\prime}}+\left[\partial_{x} g\left(y_{2}-\bar{h}\right)-\partial_{x} h\left(y_{2}-\bar{g}\right)\right] \partial_{y_{2}} .
$$

Similarly, noting that

$$
M \partial_{y}=M \partial_{y} y_{2} \partial_{y_{2}}=\partial_{y}\left(M y_{2}\right) \partial_{y_{2}}=(\bar{h}-\bar{g}) \partial_{y_{2}},
$$

we find that

$$
M \partial_{y}=(\bar{h}-\bar{g}) \partial_{y_{2}}
$$




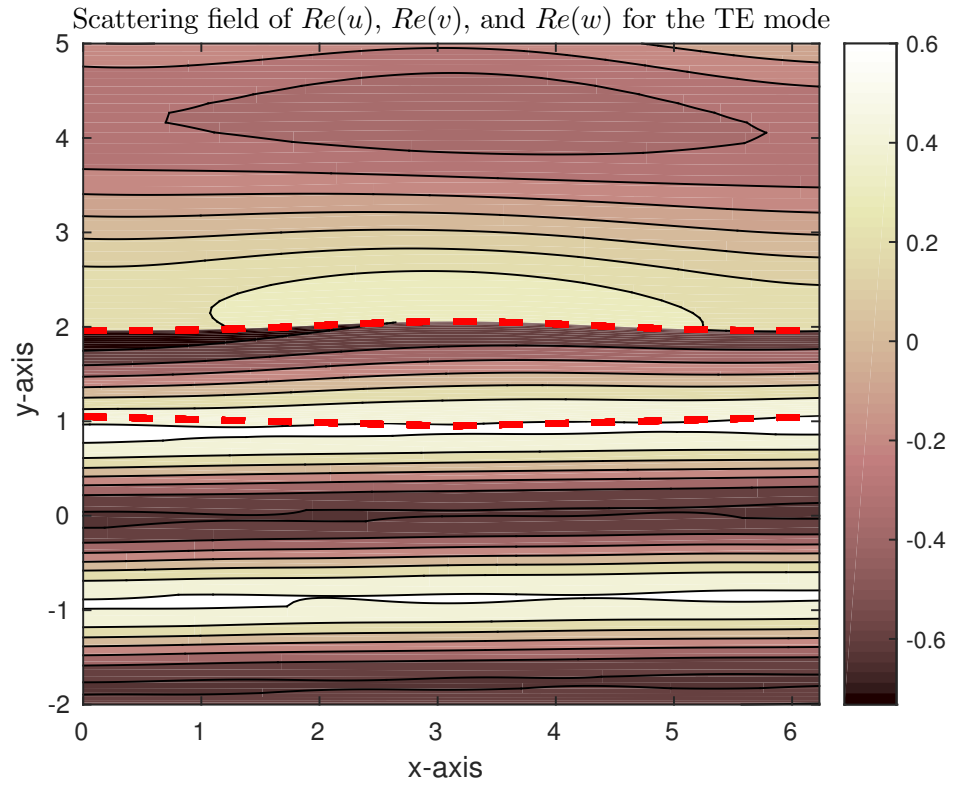

Figure 15: Plot of the real part of the scattered field for rough interface configuration (13): TE mode. Layer interfaces depicted in dashed red lines.

For $i=1,3$, setting

$$
C_{i}=\left[\xi_{i}-\left(\bar{\varphi}_{i}+\varphi_{i}\right)\right], \quad D_{i}=\frac{\partial \varphi_{i}}{\partial x^{\prime}}\left(y_{i}-\xi_{i}\right), \quad E_{i}=\left(\xi_{i}-\bar{\varphi}_{i}\right),
$$

and

$$
C_{2}=M, \quad D_{2}=\partial_{x} g\left(y_{2}-\bar{h}\right)-\partial_{x} h\left(y_{2}-\bar{g}\right), \quad E_{2}=\bar{h}-\bar{g},
$$

we rewrite (A.1) as

$$
C_{i} \partial_{x}=C_{i} \partial_{x^{\prime}}+D_{i} \partial_{y_{i}}, \quad C_{i} \partial_{y}=E_{i} \partial_{y_{i}}, \quad i=1,2,3 .
$$

We now seek forms for the second derivatives

$$
\begin{aligned}
& C^{2} \partial_{x}^{2}=C \partial_{x}\left(C \partial_{x}\right)-C\left(\partial_{x} C\right) \partial_{x} \\
& C^{2} \partial_{y}^{2}=C \partial_{y_{i}}\left(C \partial_{y_{i}}\right)-C\left(\partial_{y_{i}} C\right) \partial_{y_{i}}
\end{aligned}
$$




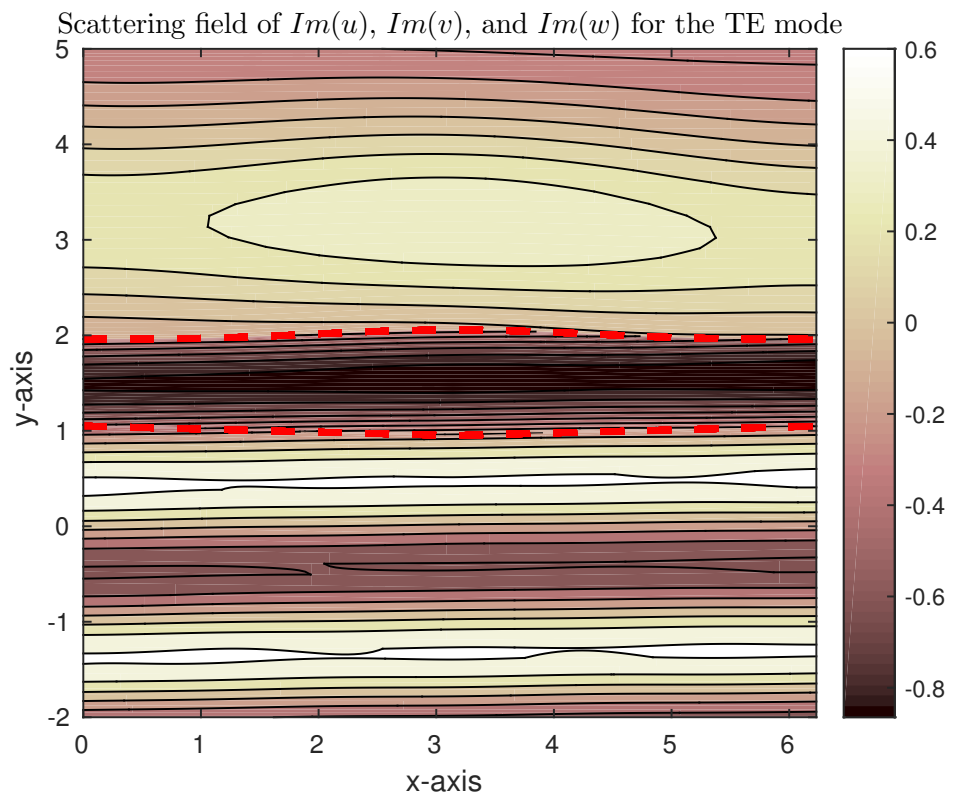

Figure 16: Plot of the imaginary part of the scattered field for rough interface configuration (13): TE mode. Layer interfaces depicted in dashed red lines.

We note that

$$
\begin{aligned}
C \partial_{x}\left(C \partial_{x}\right)= & {\left[C \partial_{x^{\prime}}+D \partial_{y_{i}}\right]\left[C \partial_{x^{\prime}}+D \partial_{y_{i}}\right] } \\
= & C \partial_{x^{\prime}}\left[C \partial_{x^{\prime}}\right]+D \partial_{y_{i}}\left[C \partial_{x^{\prime}}\right]+C \partial_{x^{\prime}}\left[D \partial_{y_{i}}\right]+D \partial_{y_{i}}\left[D \partial_{y_{i}}\right] \\
= & \partial_{x^{\prime}}\left[C^{2} \partial_{x^{\prime}}\right]-\left(\partial_{x^{\prime}} C\right)\left[C \partial_{x^{\prime}}\right]+\partial_{y_{i}}\left[C D \partial_{x^{\prime}}\right]-\left(\partial_{y_{i}} D\right)\left[C \partial_{x^{\prime}}\right] \\
& +\partial_{x^{\prime}}\left[C D \partial_{y_{i}}\right]-\left(\partial_{x^{\prime}} C\right)\left[D \partial_{y_{i}}\right]+\partial_{y_{i}}\left[D^{2} \partial_{y_{i}}\right]-\left(\partial_{y_{i}} D\right)\left[D \partial_{y_{i}}\right],
\end{aligned}
$$

and

$$
C\left(\partial_{x} C\right) \partial_{x}=\left(\partial_{x^{\prime}} C\right)\left[C \partial_{x^{\prime}}+D \partial_{y_{i}}\right]=\left(\partial_{x^{\prime}} C\right)\left[C \partial_{x^{\prime}}\right]+\left(\partial_{x^{\prime}} C\right)\left[D \partial_{y_{i}}\right],
$$

and

$$
C^{2} \partial_{y}^{2}=C \partial_{y}\left(C \partial_{y}\right)-C\left(\partial_{y} C\right) \partial_{y}=E^{2} \partial_{y_{i}}^{2} .
$$

Hence, we deduce that the Laplacian transforms as

$$
\begin{aligned}
C^{2} \Delta= & \partial_{x^{\prime}}\left[C^{2} \partial_{x^{\prime}}\right]-\left(\partial_{x^{\prime}} C\right)\left[C \partial_{x^{\prime}}\right]+\partial_{y_{i}}\left[C D \partial_{x^{\prime}}\right]-\left(\partial_{y_{i}} D\right)\left[C \partial_{x^{\prime}}\right] \\
& +\partial_{x^{\prime}}\left[C D \partial_{y_{i}}\right]-\left(\partial_{x^{\prime}} C\right)\left[D \partial_{y_{i}}\right]+\partial_{y_{i}}\left[D^{2} \partial_{y_{i}}\right]-\left(\partial_{y_{i}} D\right)\left[D \partial_{y_{i}}\right] \\
& -\left(\partial_{x^{\prime}} C\right)\left[C \partial_{x^{\prime}}\right]-\left(\partial_{x^{\prime}} C\right)\left[D \partial_{y_{i}}\right]+E^{2} \partial_{y_{i}}^{2}
\end{aligned}
$$




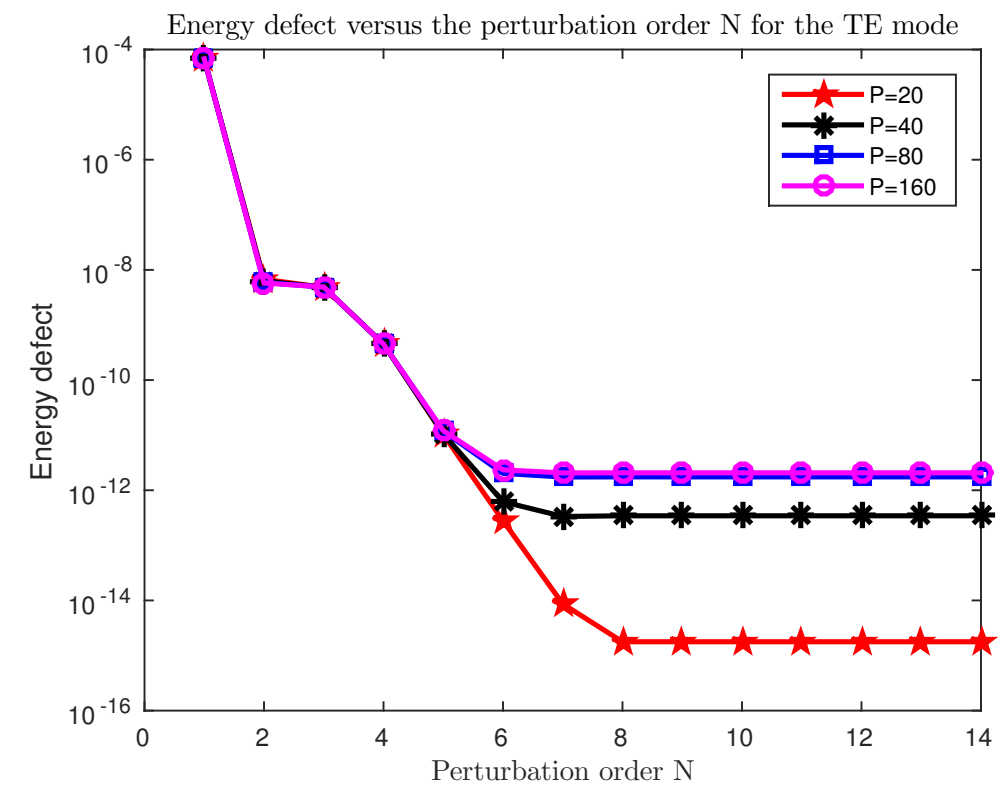

Figure 17: Energy defect versus perturbation order, $N$, for rough interface configuration (13) with $P=20,40,80,160$ : TE mode.

Noting that $\partial_{x^{\prime}} C=-\partial_{y_{i}} D$, we derive that solutions of the Helmholtz equation satisfy

$$
\begin{aligned}
0= & C^{2} \Delta u_{i}+C^{2} k^{2} u_{i} \\
= & \partial_{x^{\prime}}\left[C^{2} \partial_{x^{\prime}} u_{i}\right]+\partial_{y_{i}}\left[C D \partial_{x^{\prime}} u_{i}\right]+\partial_{x^{\prime}}\left[C D \partial_{y_{i}} u_{i}\right]-\left(\partial_{x^{\prime}} C\right)\left[D \partial_{y_{i}} u_{i}\right] \\
& +\partial_{y_{i}}\left[D^{2} \partial_{y_{i}} u_{i}\right]-\left(\partial_{x^{\prime}} C\right)\left[C \partial_{x^{\prime}} u_{i}\right]+E^{2} \partial_{y_{i}}^{2} u_{i}+C^{2} k^{2} u_{i} .
\end{aligned}
$$

Setting $C^{2}(x)=E^{2}+F(x)$, we find that

$$
\begin{array}{r}
\partial_{x^{\prime}}\left[E^{2} \partial_{x^{\prime}} u_{i}\right]+E^{2} \partial_{y_{i}}^{2} u_{i}+E^{2} k^{2} u_{i}=-\partial_{x^{\prime}}\left[F \partial_{x^{\prime}} u_{i}\right]-k^{2} F u_{i}-\partial_{y_{i}}\left[C D \partial_{x^{\prime}} u_{i}\right] \\
-\partial_{x^{\prime}}\left[C D \partial_{y_{i}} u_{i}\right]+\left(\partial_{x^{\prime}} C\right)\left[D \partial_{y_{i}} u_{i}\right]-\partial_{y_{i}}\left[D^{2} \partial_{y_{i}} u_{i}\right]+\left(\partial_{x^{\prime}} C\right)\left[C \partial_{x^{\prime}} u_{i}\right]
\end{array}
$$




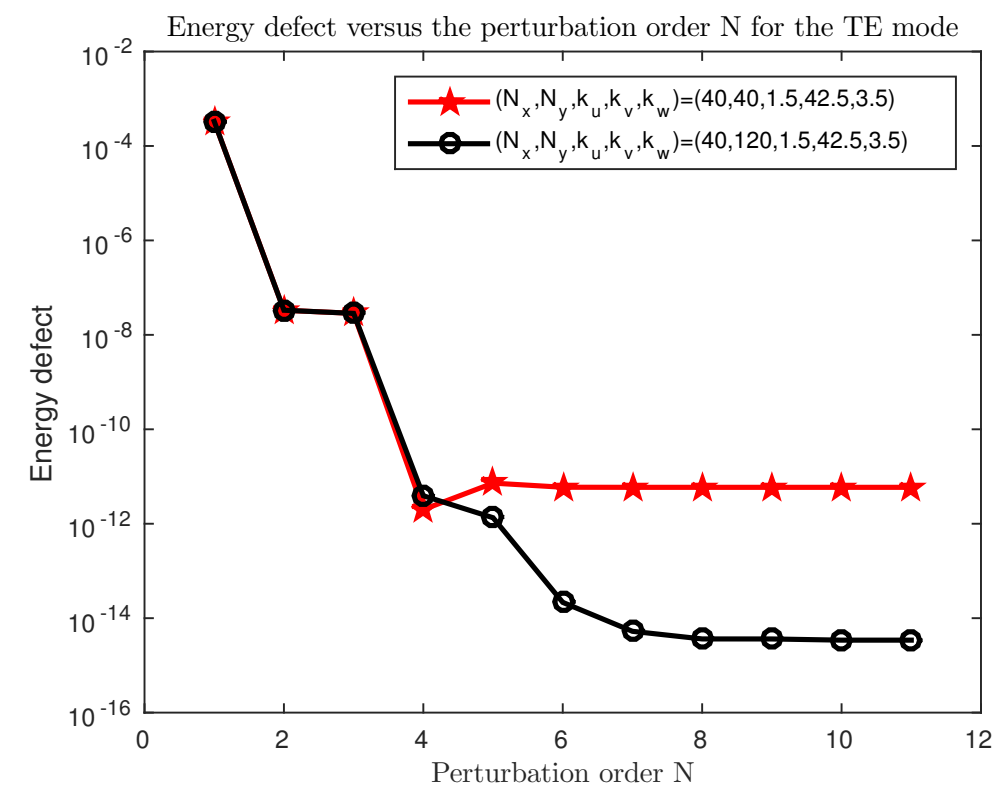

Figure 18: Energy defect versus perturbation order, $N$, for smooth interface configuration (12) with $k_{v}=42.5$ : TE mode.

and hence we arrive at

$$
\begin{aligned}
\partial_{x^{\prime}}^{2} u_{i}+\partial_{y_{i}}^{2} u_{i}+k^{2} u_{i}= & \frac{1}{E^{2}} \partial_{x^{\prime}}\left[-F \partial_{x^{\prime}} u_{i}-C D \partial_{y_{i}} u_{i}\right] \\
+ & \frac{1}{E^{2}} \partial_{y_{i}}\left[-C D \partial_{x^{\prime}} u_{i}-D^{2} \partial_{y_{i}} u_{i}\right] \\
& +\frac{1}{E^{2}}\left[-k^{2} F u_{i}+\left(\partial_{x^{\prime}} C\right)\left[D \partial_{y_{i}} u_{i}+C \partial_{x^{\prime}} u_{i}\right]\right] .
\end{aligned}
$$

Anticipating our boundary perturbation approach we introduce $\varepsilon$ as specified in Remark 1 and rearrange the right hand side of (A.2). For $i=1,3$ we have

$$
\begin{aligned}
& C_{i}=\xi_{i}-\left(\bar{\varphi}_{i}+\varepsilon \psi_{i}\right), \quad D_{i}=\varepsilon \partial_{x^{\prime}} \psi_{i}\left(y_{i}-\xi_{i}\right), \\
& E_{i}=\xi_{i}-\bar{\varphi}_{i}, \quad F_{i}=\varepsilon^{2} \psi_{i}^{2}+\varepsilon \psi_{i}\left(2 \bar{\varphi}_{i}-2 \xi_{i}\right) .
\end{aligned}
$$

Hence, (A.2) becomes

$$
\partial_{x^{\prime}}^{2} u_{i}+\partial_{y_{i}}^{2} u_{i}+k^{2} u_{i}=\frac{1}{E_{i}^{2}}\left[\partial_{x^{\prime}} R_{i}^{x}+\partial_{y_{i}} R_{i}^{y}+R_{i}\right]
$$


where

$$
\begin{aligned}
R_{i}^{x}= & -F_{i} \partial_{x^{\prime}} u_{i}-C_{i} D_{i} \partial_{y_{i}} u_{i} \\
= & -\left(\varepsilon^{2} \psi_{i}^{2}+\varepsilon \psi_{i}\left(2 \bar{\varphi}_{i}-2 \xi_{i}\right)\right) \partial_{x^{\prime}} u_{i}-\left(\xi-\bar{\varphi}_{i}-\varepsilon \psi_{i}\right)\left(\varepsilon \partial_{x^{\prime}} \psi_{i}\right)\left(y_{i}-\xi_{i}\right) \partial_{y_{i}} u_{i} \\
= & -\varepsilon \psi_{i}\left(2 \bar{\varphi}_{i}-2 \xi_{i}\right) \partial_{x^{\prime}} u_{i}-\varepsilon\left(\xi_{i}-\bar{\varphi}_{i}\right) \partial_{x^{\prime}} \psi_{i}\left(y_{i}-\xi_{i}\right) \partial_{y_{i}} u_{i} \\
& -\varepsilon^{2} \psi_{i}^{2} \partial_{x^{\prime}} u_{i}+\varepsilon^{2} \psi_{i} \partial_{x^{\prime}} \psi_{i}\left(y_{i}-\xi_{i}\right) \partial_{y_{i}} u_{i}
\end{aligned}
$$

and

$$
\begin{aligned}
R_{i}^{y}= & -C_{i} D_{i} \partial_{x^{\prime}} u_{i}-D_{i}^{2} \partial_{y_{i}} u_{i} \\
= & -\left(\xi_{i}-\bar{\varphi}_{i}-\varepsilon \psi_{i}\right) \varepsilon \partial_{x^{\prime}} \psi_{i}\left(y_{i}-\xi_{i}\right) \partial_{x^{\prime}} u_{i}-\varepsilon^{2}\left(\partial_{x^{\prime}} \psi_{i}\right)^{2}\left(y_{i}-\xi_{i}\right)^{2} \partial_{y_{i}} u_{i} \\
= & -\varepsilon\left(\xi_{i}-\bar{\varphi}_{i}\right) \partial_{x^{\prime}} \psi_{i}\left(y_{i}-\xi_{i}\right) \partial_{x^{\prime}} u_{i}+\varepsilon^{2} \psi_{i} \partial_{x^{\prime}} \psi_{i}\left(y_{i}-\xi_{i}\right) \partial_{x^{\prime}} u_{i} \\
& -\varepsilon^{2}\left(\partial_{x^{\prime}} \psi_{i}\right)^{2}\left(y_{i}-\xi_{i}\right)^{2} \partial_{y_{i}} u_{i},
\end{aligned}
$$

and

$$
\begin{aligned}
R_{i}= & -k^{2} F_{i} u_{i}+\left(\partial_{x^{\prime}} C_{i}\right)\left[D_{i} \partial_{y_{i}} u_{i}+C_{i} \partial_{x^{\prime}} u_{i}\right] \\
= & -k^{2} \varepsilon \psi_{i}\left(\varepsilon \psi_{i}+2 \bar{\varphi}_{i}-2 \xi_{i}\right) u_{i}-\varepsilon \partial_{x^{\prime}} \psi_{i}\left[\varepsilon \partial_{x^{\prime}} \psi_{i}\left(y_{i}-\xi_{i}\right) \partial_{y_{i}} u_{i}\right. \\
& \left.+\left(\xi_{i}-\bar{\varphi}_{i}-\varepsilon \psi_{i}\right) \partial_{x^{\prime}} u_{i}\right] \\
= & -\varepsilon k^{2} \psi_{i}\left(2 \bar{\varphi}_{i}-2 \xi_{i}\right) u_{i}-\varepsilon \partial_{x^{\prime}} \psi_{i}\left(\xi_{i}-\bar{\varphi}\right) \partial_{x^{\prime}} u_{i}-\varepsilon^{2} k^{2}\left(\psi_{i}\right)^{2} u_{i} \\
& -\varepsilon^{2}\left(\partial_{x^{\prime}} \psi_{i}\right)^{2}\left(y_{i}-\xi_{i}\right) \partial_{y_{i}} u_{i}+\varepsilon^{2} \psi_{i} \partial_{x^{\prime}} \psi_{i} \partial_{x^{\prime}} u_{i} .
\end{aligned}
$$

For $i=2$ we have

$$
\begin{aligned}
& C_{2}=(\bar{h}-\bar{g})+\varepsilon\left(f_{h}-f_{g}\right), \\
& D_{2}=\varepsilon\left[\partial_{x^{\prime}} f_{g}\left(y_{2}-\bar{h}\right)-\partial_{x^{\prime}} f_{h}\left(y_{2}-\bar{g}\right)\right], \\
& E_{2}=\bar{h}-\bar{g}, \\
& F_{2}=2 \varepsilon\left(f_{h}-f_{g}\right)(\bar{h}-\bar{g})+\varepsilon^{2}\left(f_{h}-f_{g}\right)^{2},
\end{aligned}
$$

and, for later use, we define $\tilde{D}_{2}:=\varepsilon D_{2}$. Hence, we find that

$$
\begin{aligned}
R_{2}^{x}= & -2 \varepsilon\left(f_{h}-f_{g}\right)(\bar{h}-\bar{g}) \partial_{x^{\prime}} u_{i}-\varepsilon^{2}\left(f_{h}-f_{g}\right)^{2} \partial_{x^{\prime}} u_{i} \\
& -\varepsilon(\bar{h}-\bar{g}) \tilde{D} \partial_{y_{2}} u_{i}-\varepsilon^{2}\left(f_{h}-f_{g}\right) \tilde{D} \partial_{y_{2}} u_{i},
\end{aligned}
$$

and

$$
R_{2}^{y}=-\varepsilon(\bar{h}-\bar{g}) \tilde{D}_{2} \partial_{x^{\prime}} u_{i}-\varepsilon^{2}\left(f_{h}-f_{g}\right) \tilde{D}_{2} \partial_{x^{\prime}} u_{i}-\varepsilon^{2} \tilde{D}^{2} \partial_{y_{2}} u_{i}
$$


and

$$
\begin{aligned}
R_{2}= & -2 \varepsilon k^{2}\left(f_{h}-f_{g}\right)(\bar{h}-\bar{g}) u_{i}-\varepsilon^{2} k^{2}\left(f_{h}-f_{g}\right)^{2} u_{i}+\varepsilon^{2} \partial_{x^{\prime}}\left(f_{h}-f_{g}\right) \tilde{D} \partial_{y_{2}} u_{i} \\
& +\varepsilon \partial_{x^{\prime}}\left(f_{h}-f_{g}\right)(\bar{h}-\bar{g}) \partial_{x^{\prime}} u_{i}+\varepsilon^{2}\left(f_{h}-f_{g}\right) \partial_{x^{\prime}}\left(f_{h}-f_{g}\right) \partial_{x^{\prime}} u_{i} .
\end{aligned}
$$

237

For the boundary conditions we recall that

$$
u^{i n c}(x, y)=e^{\mathrm{i} \alpha x-\mathrm{i} \beta^{u} y},
$$

and write $(2 \mathrm{~d})-(2 \mathrm{i})$ as

$$
\begin{array}{ll}
u_{1}-u_{2}=-e^{\mathrm{i} \alpha x-\mathrm{i} \beta^{u}(\bar{g}+g(x))}, & y=\bar{g}+g(x), \\
u_{2}-u_{3}=0, & y=\bar{h}+h(x), \\
\partial_{N_{g}} u_{1}-\tau^{2} \partial_{N_{g}} u_{2}=\left(\mathrm{i} \alpha \partial_{x} g(x)+\mathrm{i} \beta^{u}\right) e^{\mathrm{i} \alpha x-\mathrm{i} \beta(\bar{g}+g(x))}, & y=\bar{g}+g(x), \\
\partial_{N_{h}} u_{2}-\sigma^{2} \partial_{N_{h}} u_{3}=0, & y=\bar{h}+h(x), \\
\partial_{y} u_{1}-T_{1}\left[u_{1}\right]=0, & y=a, \\
\partial_{y} u_{3}-T_{3}\left[u_{3}\right]=0, & y=b .
\end{array}
$$

At $y=a$ and $y=b$, we find that

$$
\partial_{y_{i}} u_{i}-\frac{C_{i}}{E_{i}} T_{i}\left[u_{i}\right]=0,
$$

for $i=1,3$, and this implies that

$$
\partial_{y_{i}} u_{i}-T_{i}\left[u_{i}\right]=-\frac{\varphi_{i}}{\xi_{i}-\bar{\varphi}_{i}} T_{i}\left[u_{i}\right]=-\frac{\varepsilon \psi_{i}}{\xi_{i}-\bar{\varphi}_{i}} T_{i}\left[u_{i}\right]
$$

At $y=\bar{g}+g(x)$ we deduce that

$$
\partial_{N_{g}} u_{1}-\tau^{2} \partial_{N_{g}} u_{2}=\left(\mathrm{i} \alpha \partial_{x^{\prime}} g+\mathrm{i} \beta^{u}\right) e^{\mathrm{i} \alpha x-\mathrm{i} \beta^{u}(\bar{g}+g(x))}
$$

which implies that

$$
-\partial_{x^{\prime}} g \partial_{x} u_{1}+\partial_{y} u_{1}-\tau^{2}\left(-\partial_{x^{\prime}} g \partial_{x} u_{2}+\partial_{y} u_{2}\right)=\left(\mathrm{i} \alpha \partial_{x^{\prime}} g+\mathrm{i} \beta^{u}\right) e^{\mathrm{i} \alpha x-\mathrm{i} \beta^{u}(\bar{g}+g(x))} .
$$

Using the change of variables, we find that

$$
\begin{gathered}
-\varepsilon \partial_{x^{\prime}} f_{g}\left[\partial_{x^{\prime}} u_{1}+\frac{\varepsilon \tilde{D}_{1}}{C_{1}} \partial_{y_{1}} u_{1}\right]+\frac{E_{1}}{C_{1}} \partial_{y_{1}} u_{1}-\tau^{2}\left[-\varepsilon \partial_{x^{\prime}} f_{g}\left(\partial_{x^{\prime}} u_{2}+\frac{\varepsilon \tilde{D}_{2}}{C_{2}} \partial_{y_{2}} u_{2}\right)+\frac{E_{2}}{C_{2}} \partial_{y_{2}} u_{2}\right] \\
=\left(\varepsilon \mathrm{i} \alpha \partial_{x^{\prime}} f_{g}+\mathrm{i} \beta^{u}\right) e^{\mathrm{i} \alpha x-\mathrm{i} \beta^{u}(\bar{g}+g(x))} .
\end{gathered}
$$


Then, we obtain that

$$
\frac{E_{1}}{C_{1}} \partial_{y_{1}} u_{1}-\tau^{2} \frac{E_{2}}{C_{2}} \partial_{y_{2}} u_{2}=\mathcal{J}_{1}^{\prime}
$$

where

$$
\begin{array}{r}
\mathcal{J}_{1}^{\prime}:=\varepsilon\left(\partial_{x^{\prime}} f_{g}\right)\left(\partial_{x^{\prime}} u_{1}\right)+\varepsilon^{2}\left(\partial_{x^{\prime}} f_{g}\right) \frac{\tilde{D}_{1}}{C_{1}} \partial_{y_{1}} u_{1}-\varepsilon \tau^{2} \partial_{x^{\prime}} f_{g} \partial_{x^{\prime}} u_{2}-\varepsilon^{2} \tau^{2} \partial_{x^{\prime}} f_{g} \frac{\tilde{D}_{2}}{C_{2}} \partial_{y_{2}} u_{2} \\
+\left(\varepsilon \mathrm{i} \alpha \partial_{x^{\prime}} f_{g}+\mathrm{i} \beta^{u}\right) e^{\mathrm{i} \alpha x-\mathrm{i} \beta^{u} \bar{g}} e^{-\mathrm{i} \beta^{u} g(x)} .
\end{array}
$$

Noting that

$$
\begin{aligned}
& E_{1} C_{2}=(a-\bar{g})(\bar{h}-\bar{g}+h-g)=(a-\bar{g})(\bar{h}-\bar{g})+(a-\bar{g})(h-g), \\
& E_{2} C_{1}=(\bar{h}-\bar{g})(a-\bar{g}-g)=(a-\bar{g})(\bar{h}-\bar{g})-g(\bar{h}-\bar{g}),
\end{aligned}
$$

we deduce that

$$
\partial_{y_{1}} u_{1}-\tau^{2} \partial_{y_{2}} u_{2}=\mathcal{J}_{1}
$$

where

$$
\mathcal{J}_{1}=\frac{C_{1} C_{2} \mathcal{J}_{1}^{\prime}-\varepsilon(a-\bar{g})\left(f_{h}-f_{g}\right) \partial_{y_{1}} u_{1}-\varepsilon \tau^{2} f_{g}(\bar{h}-\bar{g}) \partial_{y_{2}} u_{2}}{(a-\bar{g})(\bar{h}-\bar{g})} .
$$

For later computations, the term $C_{1} C_{2} \mathcal{J}_{1}^{\prime}$ can be written

$C_{1} C_{2} \mathcal{J}_{1}^{\prime}=(a-\bar{g})(\bar{h}-\bar{g}) \mathcal{J}_{1}^{\prime}+\varepsilon \mathcal{J}_{1}^{\prime}\left((a-\bar{g})\left(f_{h}-f_{g}\right)-f_{g}(\bar{h}-\bar{g})\right)-\varepsilon^{2} f_{g}\left(f_{h}-f_{g}\right) \mathcal{J}_{1}^{\prime}$.

Similarly, at $y=\bar{h}+h(x)$, we find that

$$
\partial_{N_{h}} u_{2}-\sigma^{2} \partial_{N_{h}} u_{3}=0
$$

which implies that

$$
\left(-\partial_{x^{\prime}} h \partial_{x} u_{2}+\partial_{y} u_{2}\right)-\sigma^{2}\left(-\partial_{x^{\prime}} h \partial_{x} u_{3}+\partial_{y} u_{3}\right)=0 .
$$

By the change of variables, we deduce that

$$
\begin{aligned}
& -\varepsilon \partial_{x^{\prime}} f_{h}\left[\partial_{x^{\prime}} u_{2}+\frac{\varepsilon \tilde{D}_{2}}{C_{2}} \partial_{y_{2}} u_{2}\right]+\frac{E_{2}}{C_{2}} \partial_{y_{2}} u_{2} \\
& \quad-\sigma^{2}\left[-\varepsilon \partial_{x^{\prime}} f_{h}\left(\partial_{x^{\prime}} u_{3}+\frac{\varepsilon \tilde{D}_{3}}{C_{3}} \partial_{y_{3}} u_{3}\right)+\frac{E_{3}}{C_{3}} \partial_{y_{3}} u_{3}\right]=0
\end{aligned}
$$


Rearranging the equations, we obtain that

$$
\frac{E_{2}}{C_{2}} \partial_{y_{2}} u_{2}-\sigma^{2} \frac{E_{3}}{C_{3}} \partial_{y_{3}} u_{3}=\mathcal{J}_{2}^{\prime}
$$

where

$$
\mathcal{J}_{2}^{\prime}=\varepsilon\left(\partial_{x^{\prime}} f_{h}\right)\left(\partial_{x^{\prime}} u_{2}\right)+\varepsilon^{2} \partial_{x^{\prime}} f_{h} \frac{\tilde{D}_{2}}{C_{2}} \partial_{y_{2}} u_{2}-\varepsilon \sigma^{2} \partial_{x^{\prime}} f_{h} \partial_{x^{\prime}} u_{3}-\varepsilon^{2} \sigma^{2} \partial_{x^{\prime}} f_{h} \frac{\tilde{D}_{3}}{C_{3}} \partial_{y_{3}} u_{3} .
$$

Noting that

$$
\begin{aligned}
& E_{2} C_{3}=(\bar{h}-\bar{g})(b-\bar{h}-h)=(\bar{h}-\bar{g})(b-\bar{h})-(\bar{h}-\bar{g}) h, \\
& E_{3} C_{2}=(b-\bar{h})(\bar{h}-\bar{g}+h-g)=(b-\bar{h})(\bar{h}-\bar{g})+(b-\bar{h})(h-g),
\end{aligned}
$$

we deduce that

$$
\partial_{y_{2}} u_{2}-\sigma^{2} \partial_{y_{3}} u_{3}=\mathcal{J}_{2}
$$

where

$$
\mathcal{J}_{2}=\frac{C_{2} C_{3} \mathcal{J}_{2}^{\prime}+\varepsilon f_{h}(\bar{h}-\bar{g}) \partial_{y_{2}} u_{2}+\varepsilon \sigma^{2}(b-\bar{h})\left(f_{h}-f_{g}\right) \partial_{y_{3}} u_{3}}{(\bar{h}-\bar{g})(b-\bar{h})}
$$

For later computations, the term $C_{2} C_{3} \mathcal{J}_{2}^{\prime}$ can be written

$C_{2} C_{3} \mathcal{J}_{2}^{\prime}=(b-\bar{h})(\bar{h}-\bar{g}) \mathcal{J}_{2}^{\prime}+\varepsilon(b-\bar{h})\left(f_{h}-f_{g}\right) \mathcal{J}_{2}^{\prime}-\varepsilon f_{h}(\bar{h}-\bar{g}) \mathcal{J}_{2}^{\prime}-\varepsilon^{2} f_{h}\left(f_{h}-f_{g}\right) \mathcal{J}_{2}^{\prime}$.

As we have a boundary perturbation method in mind, it is clearly important to expand these forms in power series in $\varepsilon$. These are recorded here for completeness. For $i=1,3$,

$$
\begin{aligned}
R_{i, n}^{x}= & -\psi_{i}\left(2 \bar{\varphi}_{i}-2 \xi_{i}\right) \partial_{x^{\prime}} u_{i, n-1}-\left(\xi_{i}-\bar{\varphi}_{i}\right) \partial_{x^{\prime}} \psi_{i}\left(y_{i}-\xi_{i}\right) \partial_{y_{i}} u_{i, n-1} \\
& -\psi_{i}^{2} \partial_{x^{\prime}} u_{i, n-2}+\psi_{i} \partial_{x^{\prime}} \psi_{i}\left(y_{i}-\xi_{i}\right) \partial_{y_{i}} u_{i, n-2}, \\
R_{i, n}^{y}= & -\left(\xi_{i}-\bar{\varphi}_{i}\right) \partial_{x^{\prime}} \psi_{i}\left(y_{i}-\xi_{i}\right) \partial_{x^{\prime}} u_{i, n-1}+\psi_{i} \partial_{x^{\prime}} \psi_{i}\left(y_{i}-\xi_{i}\right) \partial_{x^{\prime}} u_{i, n-2} \\
& -\left(\partial_{x^{\prime}} \psi_{i}\right)^{2}\left(y_{i}-\xi_{i}\right)^{2} \partial_{y_{i}} u_{i, n-2}, \\
R_{i, n}= & -k^{2} \psi_{i}\left(2 \bar{\varphi}_{i}-2 \xi_{i}\right) u_{i, n-1}-\partial_{x^{\prime}} \psi_{i}\left(\xi_{i}-\bar{\varphi}\right) \partial_{x^{\prime}} u_{i, n-1}-k^{2}\left(\psi_{i}\right)^{2} u_{i, n-2} \\
& -\left(\partial_{x^{\prime}} \psi_{i}\right)^{2}\left(y_{i}-\xi_{i}\right) \partial_{y_{i}} u_{i, n-2}+\psi_{i} \partial_{x^{\prime}} \psi_{i} \partial_{x^{\prime}} u_{i, n-2},
\end{aligned}
$$


and

$$
\begin{aligned}
R_{2, n}^{x}= & -2\left(f_{h}-f_{g}\right)(\bar{h}-\bar{g}) \partial_{x^{\prime}} u_{i, n-1}-\left(f_{h}-f_{g}\right)^{2} \partial_{x^{\prime}} u_{i, n-2} \\
& -(\bar{h}-\bar{g}) \tilde{D}_{2} \partial_{y_{2}} u_{i, n-1}-\left(f_{h}-f_{g}\right) \tilde{D}_{2} \partial_{y_{2}} u_{i, n-2}, \\
R_{2, n}^{y}= & -(\bar{h}-\bar{g}) \tilde{D}_{2} \partial_{x^{\prime}} u_{i, n-1}-\left(f_{h}-f_{g}\right) \tilde{D}_{2} \partial_{x^{\prime}} u_{i, n-2}-\tilde{D}_{2}^{2} \partial_{y_{2}} u_{i, n-2}, \\
R_{2, n}= & -2 k^{2}\left(f_{h}-f_{g}\right)(\bar{h}-\bar{g}) u_{i, n-1}-k^{2}\left(f_{h}-f_{g}\right)^{2} u_{i, n-2} \\
& +\partial_{x^{\prime}}\left(f_{h}-f_{g}\right) \tilde{D}_{2} \partial_{y_{2}} u_{i, n-2} \\
& +\partial_{x^{\prime}}\left(f_{h}-f_{g}\right)(\bar{h}-\bar{g}) \partial_{x^{\prime}} u_{i, n-1}+\left(f_{h}-f_{g}\right) \partial_{x^{\prime}}\left(f_{h}-f_{g}\right) \partial_{x^{\prime}} u_{i, n-2} .
\end{aligned}
$$

For the boundary terms, we write

$$
\begin{aligned}
\mathcal{J}_{1, n} & =\frac{C_{1} C_{2} \mathcal{J}_{1, n}^{\prime}-(a-\bar{g})\left(f_{h}-f_{g}\right) \partial_{y_{1}} u_{1, n-1}-\tau^{2} f_{g}(\bar{h}-\bar{g}) \partial_{y_{2}} u_{2, n-1}}{(a-\bar{g})(\bar{h}-\bar{g})}, \\
\mathcal{J}_{2, n} & =\frac{C_{2} C_{3} \mathcal{J}_{2, n}^{\prime}+f_{h}(\bar{h}-\bar{g}) \partial_{y_{2}} u_{2, n-1}+\sigma^{2}(b-\bar{h})\left(f_{h}-f_{g}\right) \partial_{y_{3}} u_{3, n-1}}{(\bar{h}-\bar{g})(b-\bar{h})},
\end{aligned}
$$

where

$$
\begin{aligned}
\mathcal{J}_{1, n}^{\prime}:=\left(\partial_{x^{\prime}} f_{g}\right)\left(\partial_{x^{\prime}} u_{1, n-1}\right)+\left(\partial_{x^{\prime}} f_{g}\right) \frac{\tilde{D}_{1}}{C_{1}} \partial_{y_{1}} u_{1, n-2} \\
-\tau^{2} \partial_{x^{\prime}} f_{g} \partial_{x^{\prime}} u_{2, n-1}-\tau^{2} \partial_{x^{\prime}} f_{g} \frac{\tilde{D}_{2}}{C_{2}} \partial_{y_{2}} u_{2, n-2} \\
+\mathrm{i} \alpha \partial_{x^{\prime}} f_{g} e^{\mathrm{i} \alpha x^{\prime}-\mathrm{i} \beta^{u} \bar{g}} \frac{\left(-\mathrm{i} \beta^{u} f_{g}\right)^{n-1}}{(n-1) !}+\mathrm{i} \beta^{u} e^{\mathrm{i} \alpha x^{\prime}-\mathrm{i} \beta^{u} \bar{g}} \frac{\left(-\mathrm{i} \beta^{u} f_{g}\right)^{n}}{n !}
\end{aligned}
$$

and

$$
\begin{aligned}
\mathcal{J}_{2, n}^{\prime}=\left(\partial_{x^{\prime}} f_{h}\right)\left(\partial_{x^{\prime}} u_{2, n-1}\right)+\partial_{x^{\prime}} & f_{h} \\
& \frac{\tilde{D}_{2}}{C_{2}} \partial_{y_{2}} u_{2, n-2} \\
& -\sigma^{2} \partial_{x^{\prime}} f_{h} \partial_{x^{\prime}} u_{3, n-1}-\sigma^{2} \partial_{x^{\prime}} f_{h} \frac{\tilde{D}_{3}}{C_{3}} \partial_{y_{3}} u_{3, n-2},
\end{aligned}
$$

and hence

$$
\begin{aligned}
C_{1} C_{2} \mathcal{J}_{1, n}^{\prime}= & (a-\bar{g})(\bar{h}-\bar{g}) \mathcal{J}_{1, n}^{\prime}+\mathcal{J}_{1, n-1}^{\prime}\left((a-\bar{g})\left(f_{h}-f_{g}\right)-f_{g}(\bar{h}-\bar{g})\right) \\
& -f_{g}\left(f_{h}-f_{g}\right) \mathcal{J}_{1, n-2}^{\prime}, \\
C_{2} C_{3} \mathcal{J}_{2, n}^{\prime}= & (b-\bar{h})(\bar{h}-\bar{g}) \mathcal{J}_{2, n}^{\prime}+(b-\bar{h})\left(f_{h}-f_{g}\right) \mathcal{J}_{2, n-1}^{\prime}-f_{h}(\bar{h}-\bar{g}) \mathcal{J}_{2, n-1}^{\prime} \\
& -f_{h}\left(f_{h}-f_{g}\right) \mathcal{J}_{2, n-2}^{\prime} .
\end{aligned}
$$


[1] J. Virieux, S. Operto, An overview of full-waveform inversion in exploration geophysics, Geophysics 74 (6) (2009) WCC1-WCC26.

[2] F. Bleibinhaus, S. Rondenay, Effects of surface scattering in fullwaveform inversion, Geophysics 74 (6) (2009) WCC69-WCC77.

[3] C. Godrèche (Ed.), Solids far from equilibrium, Cambridge University Press, Cambridge, 1992.

[4] F. Natterer, F. Wübbeling, Mathematical methods in image reconstruction, SIAM Monographs on Mathematical Modeling and Computation, Society for Industrial and Applied Mathematics (SIAM), Philadelphia, PA, 2001.

[5] L. M. Brekhovskikh, Y. P. Lysanov, Fundamentals of Ocean Acoustics, Springer-Verlag, Berlin, 1982.

[6] H. Raether, Surface plasmons on smooth and rough surfaces and on gratings, Springer, Berlin, 1988.

[7] S. A. Maier, Plasmonics: Fundamentals and Applications, Springer, New York, 2007.

[8] S. Enoch, N. Bonod, Plasmonics: From Basics to Advanced Topics, Springer Series in Optical Sciences, Springer, New York, 2012.

[9] T. W. Ebbesen, H. J. Lezec, H. F. Ghaemi, T. Thio, P. A. Wolff, Extraordinary optical transmission through sub-wavelength hole arrays, Nature 391 (6668) (1998) 667-669.

[10] M. Moskovits, Surface-enhanced spectroscopy, Reviews of Modern Physics 57 (3) (1985) 783-826.

[11] J. Homola, Surface plasmon resonance sensors for detection of chemical and biological species, Chemical Reviews 108 (2) (2008) 462-493.

[12] H. Im, S. H. Lee, N. J. Wittenberg, T. W. Johnson, N. C. Lindquist, P. Nagpal, D. J. Norris, S.-H. Oh, Template-stripped smooth Ag nanohole arrays with silica shells for surface plasmon resonance biosensing, ACS Nano 5 (2011) 6244-6253. 
[13] N. C. Lindquist, T. W. Johnson, J. Jose, L. M. Otto, S.-H. Oh, Ultrasmooth metallic films with buried nanostructures for backside reflectionmode plasmonic biosensing, Annalen der Physik 524 (2012) 687-696.

[14] J. Jose, L. R. Jordan, T. W. Johnson, S. H. Lee, N. J. Wittenberg, S.H. Oh, Topographically flat substrates with embedded nanoplasmonic devices for biosensing, Adv Funct Mater 23 (2013) 2812-2820.

[15] F. Reitich, T. W. Johnson, S.-H. Oh, G. Meyer, A fast and high-order accurate boundary perturbation method for characterization and design in nanoplasmonics, Journal of the Optical Society of America, A 30 (2013) 2175-2187.

[16] D. P. Nicholls, F. Reitich, T. W. Johnson, S.-H. Oh, Fast high-order perturbation of surfaces (HOPS) methods for simulation of multi-layer plasmonic devices and metamaterials, Journal of the Optical Society of America, A 31 (8) (2014) 1820-1831.

[17] D. C. Dobson, Optimal design of periodic antireflective structures for the Helmholtz equation, European J. Appl. Math. 4 (4) (1993) 321-339.

[18] D. C. Dobson, A variational method for electromagnetic diffraction in biperiodic structures, RAIRO Modél. Math. Anal. Numér. 28 (4) (1994) 419-439.

[19] G. Bao, D. C. Dobson, Nonlinear optics in periodic diffractive structures, in: Second International Conference on Mathematical and Numerical Aspects of Wave Propagation (Newark, DE, 1993), SIAM, Philadelphia, PA, 1993, pp. 30-38.

[20] G. Bao, Finite element approximation of time harmonic waves in periodic structures, SIAM J. Numer. Anal. 32 (4) (1995) 1155-1169.

[21] G. Bao, Numerical analysis of diffraction by periodic structures: TM polarization, Numer. Math. 75 (1) (1996) 1-16.

[22] D. P. Nicholls, A high-order perturbation of surfaces (HOPS) approach to Fokas integral equations: Three-dimensional layered media scattering, Quarterly of Applied Mathematics 74 (2016) 61-87. 
[23] D. P. Nicholls, A method of field expansions for vector electromagnetic scattering by layered periodic crossed gratings, Journal of the Optical Society of America, A 32 (5) (2015) 701-709.

[24] D. P. Nicholls, S.-H. Oh, T. W. Johnson, F. Reitich, Launching surface plasmon waves via vanishingly small periodic gratings, Journal of Optical Society of America, A 33 (3) (2016) 276-285.

[25] D. P. Nicholls, Numerical solution of diffraction problems: A high-order perturbation of surfaces/asymptotic waveform evaluation method, submitted.

[26] D. Colton, R. Kress, Inverse acoustic and electromagnetic scattering theory, 2nd Edition, Springer-Verlag, Berlin, 1998.

[27] A. Barnett, L. Greengard, A new integral representation for quasiperiodic scattering problems in two dimensions, BIT Numerical Mathematics 51 (2011) 67-90.

[28] J. Lai, M. Kobayashi, A. Barnett, A fast and robust solver for the scattering from a layered periodic structure containing multi-particle inclusions, J. Comput. Phys. 298 (2015) 194-208.

[29] M. H. Cho, A. Barnett, Robust fast direct integral equation solver for quasi-periodic scattering problems with a large number of layers, Optics Express 23 (2) (2015) 1775-1799.

[30] O. Bruno, B. Delourme, Rapidly convergent two-dimensional quasiperiodic green function throughout the spectrum-including Wood anomalies, Journal of Computational Physics 262 (2014) 262-290.

[31] O. Bruno, S. Shipman, C. Turc, S. Venakides, Superalgebraically convergent smoothly windowed lattice sums for doubly periodic green functions in three-dimensional space, Proceedings of the Royal Society A (to appear).

[32] O. Bruno, M. Lyon, C. Perez-Arancibia, C. Turc, Windowed green function method for layered-media scattering, SIAM Journal on Applied Mathematics (to appear). 
[33] L. Greengard, V. Rokhlin, A fast algorithm for particle simulations, J. Comput. Phys. 73 (2) (1987) 325-348.

[34] F. Reitich, K. Tamma, State-of-the-art, trends, and directions in computational electromagnetics, CMES Comput. Model. Eng. Sci. 5 (4) (2004) 287-294.

[35] M. Moharam, T. Gaylord, Rigorous coupled-wave analysis of planargrating diffraction, Journal of the Optical Society of America 71 (1981) 811-818.

[36] R. C. Rumpf, Improved formulation of scattering matrices for semianalytical methods that is consistent with convention, Progress In Electromagnetics Research B 35 (2011) 241-261.

[37] L. Rayleigh, On the dynamical theory of gratings, Proc. Roy. Soc. London A79 (1907) 399-416.

[38] S. O. Rice, Reflection of electromagnetic waves from slightly rough surfaces, Comm. Pure Appl. Math. 4 (1951) 351-378.

[39] O. Bruno, F. Reitich, Numerical solution of diffraction problems: A method of variation of boundaries, J. Opt. Soc. Am. A 10 (6) (1993) $1168-1175$.

[40] O. Bruno, F. Reitich, Numerical solution of diffraction problems: A method of variation of boundaries. II. Finitely conducting gratings, Padé approximants, and singularities, J. Opt. Soc. Am. A 10 (11) (1993) 23072316.

[41] O. Bruno, F. Reitich, Numerical solution of diffraction problems: A method of variation of boundaries. III. Doubly periodic gratings, J. Opt. Soc. Am. A 10 (12) (1993) 2551-2562.

[42] D. P. Nicholls, F. Reitich, Shape deformations in rough surface scattering: Cancellations, conditioning, and convergence, J. Opt. Soc. Am. A 21 (4) (2004) 590-605.

[43] D. P. Nicholls, F. Reitich, Shape deformations in rough surface scattering: Improved algorithms, J. Opt. Soc. Am. A 21 (4) (2004) 606-621. 
[44] D. P. Nicholls, F. Reitich, Boundary perturbation methods for highfrequency acoustic scattering: Shallow periodic gratings, J. Acoust. Soc. Amer. 123 (5) (2008) 2531-2541.

[45] A. Malcolm, D. P. Nicholls, A field expansions method for scattering by periodic multilayered media, Journal of the Acoustical Society of America 129 (4) (2011) 1783-1793.

[46] D. P. Nicholls, F. Reitich, A new approach to analyticity of DirichletNeumann operators, Proc. Roy. Soc. Edinburgh Sect. A 131 (6) (2001) 1411-1433.

[47] D. P. Nicholls, F. Reitich, Stability of high-order perturbative methods for the computation of Dirichlet-Neumann operators, J. Comput. Phys. 170 (1) (2001) 276-298.

[48] D. P. Nicholls, F. Reitich, Analytic continuation of Dirichlet-Neumann operators, Numer. Math. 94 (1) (2003) 107-146.

[49] J. Wilkening, V. Vasan, Comparison of five methods of computing the Dirichlet-Neumann operator for the water wave problem, in: Nonlinear wave equations: analytic and computational techniques, Vol. 635 of Contemp. Math., Amer. Math. Soc., Providence, RI, 2015, pp. 175-210.

[50] B. Hu, D. P. Nicholls, Analyticity of Dirichlet-Neumann operators on Hölder and Lipschitz domains, SIAM J. Math. Anal. 37 (1) (2005) 302320 .

[51] D. P. Nicholls, J. Shen, A stable, high-order method for two-dimensional bounded-obstacle scattering, SIAM J. Sci. Comput. 28 (4) (2006) 13981419 .

[52] Q. Fang, D. P. Nicholls, J. Shen, A stable, high-order method for threedimensional bounded-obstacle scattering, J. Comput. Phys. 224 (2) (2007) 1145-1169.

[53] D. P. Nicholls, J. Shen, A rigorous numerical analysis of the transformed field expansion method, SIAM Journal on Numerical Analysis 47 (4) (2009) 2708-2734. 
[54] Y. He, D. P. Nicholls, J. Shen, An efficient and stable spectral method for electromagnetic scattering from a layered periodic structure, Journal of Computational Physics 231 (8) (2012) 3007-3022.

[55] H. D. Han, X. N. Wu, Approximation of infinite boundary condition and its application to finite element methods, J. Comput. Math. 3 (2) (1985) 179-192.

[56] J. B. Keller, D. Givoli, Exact nonreflecting boundary conditions, J. Comput. Phys. 82 (1) (1989) 172-192.

[57] D. Givoli, Nonreflecting boundary conditions, J. Comput. Phys. 94 (1) (1991) 1-29.

[58] D. Givoli, J. B. Keller, Special finite elements for use with high-order boundary conditions, Comput. Methods Appl. Mech. Engrg. 119 (3-4) (1994) 199-213.

[59] D. Givoli, Numerical methods for problems in infinite domains, Vol. 33 of Studies in Applied Mechanics, Elsevier Scientific Publishing Co., Amsterdam, 1992.

[60] M. J. Grote, J. B. Keller, On nonreflecting boundary conditions, J. Comput. Phys. 122 (2) (1995) 231-243.

[61] D. Givoli, Recent advances in the DtN FE method, Arch. Comput. Methods Engrg. 6 (2) (1999) 71-116.

[62] D. P. Nicholls, N. Nigam, Exact non-reflecting boundary conditions on general domains, J. Comput. Phys. 194 (1) (2004) 278-303.

[63] D. P. Nicholls, N. Nigam, Error analysis of a coupled finite element/DtN map algorithm on general domains, Numer. Math. 105 (2) (2006) 267298.

[64] L. Chindelevitch, D. P. Nicholls, N. Nigam, Error analysis and preconditioning for an enhanced DtN-FE algorithm for exterior scattering problems, J. Comput. App. Math. 204 (2007) 493-504.

[65] R. Petit (Ed.), Electromagnetic theory of gratings, Springer-Verlag, Berlin, 1980. 
[66] Y. He, M. Min, D. P. Nicholls, A spectral element method with transparent boundary condition for periodic layered media scattering, Journal of Scientific Computing (to appear).

[67] N. A. Phillips, A coordinate system having some special advantages for numerical forecasting, Journal of the Atmospheric Sciences 14 (2) (1957) 184-185.

[68] J. Chandezon, D. Maystre, G. Raoult, A new theoretical method for diffraction gratings and its numerical application, J. Opt. 11 (7) (1980) 235-241.

[69] J. Shen, Efficient spectral-Galerkin method. I. Direct solvers of secondand fourth-order equations using Legendre polynomials, SIAM J. Sci. Comput. 15 (6) (1994) 1489-1505.

[70] J. Shen, T. Tang, L.-L. Wang, Spectral methods, Vol. 41 of Springer Series in Computational Mathematics, Springer, Heidelberg, 2011, algorithms, analysis and applications. 